\title{
Ionosphere-thermosphere Relation as Seen in Measured and Modeled Electron and Neutral Densities
}

Kristin Vielberg ( $\square$ vielberg@geod.uni-bonn.de)

Rheinische Friedrich-Wilhelms-Universitat Bonn https://orcid.org/0000-0001-5429-0988

\section{Armin Corbin}

Rheinische Friedrich-Wilhelms-Universitat Bonn

Jürgen Kusche

Rheinische Friedrich-Wilhelms-Universitat Bonn

Chao Xiong

Wuhan University

Claudia Stolle

GFZ: Deutsches Geoforschungszentrum Potsdam

Full paper

Keywords: Neutral density, Electron density, in-situ data, TIE-GCM, GRACE, CHAMP, correlation, thermosphere, ionosphere

Posted Date: May 7th, 2021

DOI: https://doi.org/10.21203/rs.3.rs-459456/v1

License: (c) (i) This work is licensed under a Creative Commons Attribution 4.0 International License. Read Full License 


\section{Ionosphere-thermosphere relation as seen in measured and mod-}

\section{eled electron and neutral densities}

3 Kristin Vielberg, Institute of Geodesy and Geoinformation, University of Bonn, Nussallee 17, 53115 Bonn, Germany, vielberg@geod.uni-bonn.de Armin Corbin, Institute of Geodesy and Geoinformation, University of Bonn, Nussallee 17, 53115 Bonn, Germany Jürgen Kusche, Institute of Geodesy and Geoinformation, University of Bonn, Nussallee 17, 53115 Bonn, Germany

Chao Xiong, Department of Space Physics, College of Electronic Information, Wuhan University, 430072 Wuhan, China

Claudia Stolle, GFZ German Research Centre for Geosciences, Telegrafenberg, 14473 Potsdam, Germany 


\section{${ }_{5}$ Abstract}

6 The availability of in-situ neutral and electron densities along the orbit of the satellite missions GRACE

7 and CHAMP provide a good opportunity to study the ionosphere-thermosphere (IT) system. The aim

8 of this paper is (1) to use these data sets, to study the IT density relation empirically via correlation

9 properties for different conditions depending on solar activity, geomagnetic latitude, and local time and

10 (2) to verify whether these relations are consistent with the output of the TIE-GCM model of the

11 thermosphere and ionosphere.

12 Our results show that the correlations of electron and neutral densities strongly depend on magnetic

13 local time (MLT) with a minimal correlation between 6-9h MLT, e.g., every 131 days for CHAMP

14 around $400 \mathrm{~km}$ altitude and every 160 days for GRACE around $500 \mathrm{~km}$. During low solar activity, the

15 correlation of modeled and measured densities agrees well for both satellites. On the contrary, we note

16 that the correlations between the modelled values are higher, especially during high solar activity,

17 where the difference between correlations of modeled and measured densities is about 0.2 . We suggest

18 that the reason for this misalignment might be related to the poor representation of the equatorial

19 density anomaly in the model especially during high solar activity.

20 We believe our results will be useful for studies that aim at assimilating electron densities into a

${ }_{21}$ physical model to improve the prediction of neutral densities, since the skill of data assimilation

22 depends to a large extent on the representation of the correlation between both densities.

\section{Keywords}

${ }_{24}$ Neutral density, Electron density, in-situ data, TIE-GCM, GRACE, CHAMP, correlation, thermosphere, 25 ionosphere

\section{Introduction}

${ }_{27}$ Although the magnitude of the neutral density at the altitude of low-Earth orbiting satellites is only ${ }_{28} 10^{-13} \mathrm{~kg} \mathrm{~m}^{-3}$ at $400 \mathrm{~km}$, it causes a considerable drag on the satellite. Therefore, applications such 29 as satellite altitude and lifetime predictions, the propagation of space debris, collision prediction or 30 avoidance, and precise orbit determination require accurate information on the neutral density, which is 
31

32 The neutral mass density in the Earth's atmosphere is made up by many constituents, e.g., helium,

з3 hydrogen and oxygen, and their altitude variations depend on solar radiation and magnetic forcing. The

34 resulting total neutral mass density varies at different temporal and spatial scales, e.g., it decreases nearly

35 exponentially with altitude due to aerostatic equilibrium. Additionally, sudden solar events, e.g., solar

36 flares, can cause short-term variations in the atmospheric temperature, density and ionization.

37 After integrating decades of data and extensive calibration efforts, empirical models, such as NRLMSIS2.0

38 (Emmert et al. 2020), DTM2020 (Bruinsma 2021) or JB2008 (Bowman et al. 2008), represent an average

39 state of the atmosphere derived from fitting mathematical equations to measurements (e.g., Emmert

40 2015). Most data sets considered in the model development provide integrated densities Bowman 2002,

${ }_{41}$ Pardini et al. 2010; Emmert 2015), since in-situ measurements are sparse. Estimations of physical models

42 provide another opportunity to predict neutral densities. Physical models are based on continuity, energy

43 and momentum equations of the ionosphere-thermosphere (IT) system. The implementation of physical

44 models is much more complex than that of empirical models, but still simplifications of the physics

45 cannot be avoided (e.g., Schunck et al. 2012). Both kind of models require several input parameters such

46 as solar and geomagnetic indices. Emmert (2015) summarizes that physical models do not achieve higher

${ }_{47}$ prediction skills than empirical models. Since physical models are not constrained to measurements, there

48 is great potential to obtain more realistic model outputs when assimilating measurements especially with

49 good global coverage. With the availability of integrated electron densities from the global navigation

50 satellite system (GNSS), it has been proposed to use physical models that assimilate measured electron 51 density data to predict neutral density (e.g., Hajj et al. 2004; Codrescu et al. 2012). Electron content 52 observations derived from a network of ground GNSS receivers is well-suited for data assimilation because 53 they are provided as Total Electron Content (TEC) maps in regular time steps as regular grids. The 54 method of choice for data assimilation is often the ensemble Kalman filter (EnKF, Evensen 2009) since it 55 runs "embarrassingly parallel", and requires minimal modifications at source code level via frameworks 56 such as PDAF (Nerger and Hiller 2013) or DART (Anderson et al. 2009), of which the latter has been 57 used to improve TIE-GCM model predictions in (e.g., Lee et al. 2012, Matsuo et al. 2013, Kodikara and

58 Zhang 2019). Assimilating electron density is expected to nudge the model to more realistic neutral 59 density. However, predicting neutral density by use of electron density may only benefit if (1) neutral ${ }_{60}$ and electron density are well (anti-)correlated and (2) if cross-correlations are correctly represented in 
${ }_{65}$ (ensemble size and breeding) and on the couplings implemented within the model. Here we argue that, ${ }_{66}$ by implementing the ergodicity hypothesis, comparing temporal cross-correlations from directly observed

${ }_{69}$ The ionosphere is immersed in the upper atmosphere and both are largely controlled by solar radiation,

${ }_{71}$ and electrons in the ionosphere, but in the meanwhile it increases the temperature of neutral atmosphere 72 and results in larger density scale height, thus, density decreases less with increasing altitude. However, 73 the enlarged atmospheric density will increase the ion-neutral collision frequency, resulting in a decrease 74 of ionospheric plasma density. Therefore, the ionisation degree of the upper atmosphere depends on 75 the balance between enhanced EUV and atmospheric scale height. Additionally, the variability of the 76 ionosphere and upper atmosphere is associated with lower atmospheric processes, e.g., tidal and wave 77 forcing from below (Immel et al. 2006: Oberheide et al. 2006; Hagan and Forbes 2009; Häusler and Lühr 78 2009; Xiong et al. 2014: Siddiqui et al. 2018).

79 Thus, due to the mentioned phenomena we can expect that there is sufficient correlation between the so thermosphere and ionosphere for the success of assimilating measured densities into physical models, but this correlation will depend on dayside/nightside, solar/magnetic forcing, also on the timescale considered, 82 and it is unclear to what extent physical models consider all this correctly. So it is relevant to quantify 83 this correlation from data.

${ }_{84}$ In this paper, we take advantage of simultaneous measurements of both neutral and electron densities 85 from the satellite missions Gravity Recovery and Climate Experiment (GRACE, Tapley et al. 2004) and ${ }_{86}$ CHAllenging Minisatellite Payload (CHAMP, Reigber et al. 2002) flying at topside ionosphere. Neutral ${ }_{87}$ densities are derived from accelerometer measurements, whereas electron densities are derived from K${ }_{88}$ Band measurements for GRACE, and taken from the Planar Langmuir Probe for CHAMP. We argue that 89 both electron density data sets used here are 'in-situ' as compared to line-integrated TEC derived from 90 GNSS data, and thus better collocated with the accelerometer-derived densities at the satellite, which 
91 provides a good opportunity to investigate the interaction between the ionosphere and upper atmosphere 92 in terms of correlations. The aim of this paper is (1) to use these data sets, which have - to our knowledge 93 - not been compared yet, to extensively study the correlation properties for different conditions depending 94 on solar activity, geomagnetic latitude, and local time and (2) to see whether this is in line with model ${ }_{95}$ physics as simulated by the physical density model TIE-GCM. Since we cannot directly assess coupling 96 strength in this way, we instead focus on the correlation of neutral and electron densities. It allows 97 us to empirically examine the strength of the relation between the ionosphere and thermosphere in the 98 model compared to measurements and thus assess the potential for predicting neutral density via the 99 assimilation of electron densities. In the next chapter, we summarize the density data sets and model settings, which are the basis for our correlation studies presented in the result section.

101 Our experiments are limited to the data of the GRACE and CHAMP missions, however, the analyses can be applied to densities from other missions such as Swarm and GRACE Follow-On as well. The densities 112 a few kilometers.

\section{In-situ neutral density} space.

in this study are not scaled to a specific altitude, instead we take the advantage of using collocated in-situ measurements directly. Our focus is to study correlations in the view of data assimilation of measured in-situ neutral and electron densities and, eventually, predicting neutral density and drag on objects in

Our correlation analyses might be limited by the sparse spatial resolution of the in-situ data, e.g., the CHAMP satellite covers all local times within 131 days. The in-situ neutral densities are certainly affected by calibration errors, but remaining biases and scales drop out in correlation studies. Another limitation of our analyses is that the output of TIE-GCM requires extrapolation at the GRACE altitude especially during nighttime, however, the gap between the upper model layer and the altitude of the satellite is only

${ }_{5}$ The total neutral mass density can be obtained from different measurement principles. Neutral mass spectrometers have been developed since the 1960s to measure the atmospheric composition (Emmert 2015). However, up to now spectrometry data are not available for all relevant constituents at LEO altitudes, which might change in the future with measurements from missions following the proposed Daedalus concept (Sarris et al. 2020: Palmroth et al. 2021). Secondly, the mass density can be estimated 
from changes in the satellite orbit. Orbit data are available from various tracking techniques such as satellite laser ranging and are often provided as two-line element (TLE) data sets for objects back to the 1960s. The resulting densities represent average values for an orbital arc of several hours lengths, thus, their temporal resolution is limited to at most once per orbit. For spherical satellites, the estimation of the ballistic coefficient, which can be related to corrections for modeled densities, is also widely used but the accuracy and spatial resolution is limited (Bowman 2002) and the ballistic coefficient is correlated with other solved-for parameters.

Here, we derive in-situ neutral densities from non-gravitational accelerations acting on the satellite measured by space-borne accelerometers, which are limited to very few missions (mostly designated gravity field missions). In a first step, it requires a calibration of accelerometer data. We apply daily biases and constant scales derived within a precise orbit determination procedure (Vielberg et al. 2018, Sect. 3.2). Compared to Vielberg et al. (2018), gravitational background models used in the calibration are updated to the following versions: static gravity field GOCO06s (Kvas et al. 2019), the time variable gravity field ITSG2018 (Kvas et al. 2019), the atmosphere and ocean dealiasing product AOD1B RL06 (Dobslaw et al. 2017), ocean tide model FES 2014b (Carrere et al. 2015).

In the next step, non-gravitational accelerations due to the radiation pressure (RP) of the Sun and the Earth are modeled as in Vielberg et al. (2020), i.e., in addition to standard models (e.g., Knocke et al. 1988), we account for thermal reradiation at the satellite's surface and for the Earth's outgoing radiation from hourly CERES SYN1deg data (CERES Science Team 2019), which is converted to radiative fluxes by applying angular distribution models (Suttles et al. 1988, 1989). The accelerations are computed for each panel of the underlying macro-model (Bettadpur (2012) for GRACE; and the DEOS model Doornbos et al. (2009) for CHAMP) and accumulated afterwards to obtain the total acceleration acting 122 on the satellite. Removing solar and Earth radiation pressure accelerations $\mathbf{a}_{\mathrm{SRP}}$ and $\mathbf{a}_{\mathrm{ERP}}$ from calibrated accelerometer measurements $\mathbf{a}_{\text {cal }}$ yields the aerodynamic acceleration

$$
\mathbf{a}_{\mathrm{aero}}=\mathbf{a}_{\mathrm{cal}}-\mathbf{a}_{\mathrm{SRP}}-\mathbf{a}_{\mathrm{ERP}} .
$$

Finally, the thermospheric neutral densities can be obtained from rearranging the analytical equation 45 of the aerodynamic acceleration (see e.g., Doornbos 2012) for the neutral density $\rho$ and inserting the measured aerodynamic acceleration from Eq.(1) as

$$
\rho=\frac{-2 m\left(\mathbf{a}_{\mathrm{aero}}\right) \cdot \mathbf{e}_{\mathbf{1}}}{A \mathbf{C}_{\mathrm{a}}\left|\mathbf{v}_{\mathrm{rel}}\right|^{2} \cdot \mathbf{e}_{\mathbf{1}}} .
$$


In Eq. 22 , the multiplication with the unit basis vector $\mathbf{e}_{1}=[1,0,0]^{\mathrm{T}}$ indicates the use of along-track accelerations only. We apply the aerodynamic coefficient $\mathbf{C}_{a}$ following Doornbos (2012) with updated temperature and neutral species densities from the NRLMSIS 2.0 (Emmert et al. 2020). An energy accommodation coefficient of 0.93 and 0.85 is used for GRACE and CHAMP, respectively. For the processing of accelerometer-derived densities, we use the GRACE Level-1B accelerometer data (ACC1B) and reduced dynamic orbits (GNV1B) (Case et al. 2010). For CHAMP, we apply the official Level 2 accelerometer data, and reduced-dynamic orbits processed at AIUB (Prange et al. $2010 ;$ Prange 2010).

\section{In-situ electron density}

Electron densities are commonly available as integrated quantities derived from the signal propagation of tracking techniques such as GNSS networks, DORIS, or radio occultations Akmaev et al. 2011; Dettmering et al. 2014 Hernández-Pajares et al. 2017; Goss et al. 2019). The resulting slant total electron content, which is the integrated electron density along the radio link, can be mapped to the vertical. Deriving electron densities at a specific location from the partitioning of TEC data into voxels is possible through inversion techniques, however, several assumptions are required (e.g., Goss et al. 2019). Here, we take advantage of in-situ electron densities with a high temporal resolution, which are limited to a few missions. The planar Langmuir probe (PLP) on-board the CHAMP satellite provides in-situ electron densities at a temporal resolution of 15 seconds (McNamara et al. 2007) accessible in the CHAMP Level 2 data. For GRACE, Xiong et al. (2010) developed a method to obtain electron densities from K-Band inter-satellite range (KBR) measurements. The KBR system measures the dual one-way range changes between the two GRACE satellites, which provides a ionospheric correction parameter that can be further used for deriving the horizontal TEC between the two spacecraft. By further dividing by the distance between the two spacecraft, the mean electron density of the volume between the two GRACE satellites is obtained. However, there is an unknown bias in the ionospheric correction parameter, as a result the derived electron density contains also an unknown bias. The bias has been removed by comparing to the electron density measurements from ground-based incoherent scatter radars Xiong

\section{Physical model TIE-GCM}

174 In this study, we use the Thermosphere Ionosphere Electrodynamics General Circulation Model (TIE175 GCM, Quian et al. 2014), which provides a three-dimensional, non-linear representation of the coupled 176 ionopshere-thermosphere system. Output fields of the TIE-GCM are computed on a regular global grid 
at $2.5^{\circ}$ resolution in longitude and latitude at 56 pressure levels. The model integration duration is 30 seconds. An exception is the period around the Halloween storm, where the integration time is reduced to 10 seconds (27.10.2003-31.12.2003). We choose the Heelis model (Heelis et al. 1982) to compute the electric potential at high latitudes, since input data for the year 2007 are not available for the Weimer model (Weimer 2005). The solar flux is given by the $F_{10.7}$ time series adjusted to one astronomical unit. The 3-hourly $K p$-index is used to represent geomagnetic activity. A file including all control parameters is part of the supplementary material. The initial values for the model runs are computed in a spin-up phase by running TIE-GCM with fixed $K p$ and $F_{10.7}$ values for the first day of the investigated period of each year 10 times, so that the model reaches a steady state.

For the present study, we use TIE-GCM neutral and electron densities during the years 2003 and 2007.

We save both every 10 minutes, leading to 144 samples per day and grid cell. We then predict the densities at the satellite's location by linear interpolation of the gridded data. For the neutral densities the logarithmized data is used to minimize interpolation errors. Whenever a satellite is above the upper layer of TIE-GCM, we use exponential extrapolation, but the distance between the upper model layer and the satellite altitude is small.

\section{Results}

\section{Data visualisation and wavelet analyses}

Fig. 1 displays daily mean values of measured neutral and electron densities between 2002 and 2011. The measured neutral density at CHAMP altitude, which is on average $3 \times 10^{-12} \mathrm{~kg} \mathrm{~m}^{-3}$ for the shown period, is higher than for GRACE $\left(0.5 \times 10^{-12} \mathrm{~kg} \mathrm{~m}^{-3}\right)$. This is directly related to the altitude of GRACE, which is above $460 \mathrm{~km}$ for the shown period, whereas CHAMP orbits Earth below $420 \mathrm{~km}$ (Fig. 1, top) during the same period. Both satellites loose altitude due to the atmospheric drag during their lifetime, but the larger drag at lower altitudes causes CHAMP to descend faster. In comparison to neutral densities, the difference in electron density magnitude between CHAMP and GRACE altitude is smaller especially during high solar activity (Fig. 1. bottom). CHAMP electron densities decrease from $0.8 \times 10^{6} \mathrm{~cm}^{-3}$ at high solar activity during 2002 to $0.1 \times 10^{6} \mathrm{~cm}^{-3}$ in 2008. A comparison with the $F_{10.7}$ index, which is a measure of the extreme ultraviolet solar radiation at a wavelength of $10.7 \mathrm{~cm}$, shows that both electron and neutral densities decrease with decreasing solar activity until the solar minimum in 2008 (Fig. 1).

In addition to the temporal evolution of daily mean densities, we present the measured and modeled 
${ }_{233} \quad F_{10.7}$ index is 129 and 73 solar flux units, respectively. it needs about five years to provide an even coverage in season-MLT.

densities with respect to magnetic local time and geomagnetic latitude during the year 2003 along the CHAMP orbit (Fig. 2). Magnetic local time (MLT) and geomagnetic latitude (MLAT) were computed following Laundal and Richmond (2017). Between 5 and 19h MLT, we can see from the shadow function (Fig. 2, bottom), which assigns whether the satellite is located in sunlight, in the Earth's shadow or in semi-shadow, that the satellite is fully in sunlight during these times. Electron densities are small during nighttime and increase between 7 and $10 \mathrm{~h}$ MLT to values around $1.2 \times 10^{6} \mathrm{~cm}^{-3}$ at daytime, whereas neutral densities at daytime are up to two times larger than during nighttime with values around $5 \times 10^{-12} \mathrm{~kg} \mathrm{~m}^{-3}$. Vertical strips in the MLT distribution corresponding to large neutral and electron densities could be caused by the uneven season-MLT coverage of the 1-year data used here. For CHAMP,

Complementary to the results above in the time domain, we apply a wavelet analysis to compare the densities in the frequency domain. We use the complex Morlet wavelet with bandwidth parameter equal to one and a central frequency of $1.5 \frac{1}{\text { day }}$. The continuous wavelet transform amplitude spectra of both neutral and electron densities from the CHAMP mission in the year 2003 (Fig. 3) show peaks at the orbital revolution of around 15 times per day and its higher harmonics. It is striking that the spectrum of the electron densities appears much smoother, which means that variations have less amplitude compared to the background. The neutral density spectrum has high amplitudes around the strong solar storm on October 29-31, 2003, and a little later during the storm on November 20. In comparison to the neutral density spectrum, the solar storm in October 2003 seems to be less distinct in the electron density spectrum, which does not mean that storms have a minor impact on electron densities since dramatic ionospheric disturbances have been seen during this storm for CHAMP (Yizengaw et al. 2006). The amplitude spectra of modeled densities (not shown here) look similar.

For further analyses, we focus on two specific years with high and low solar activity, respectively. Since electron density data for CHAMP are sparse in 2008, we choose the year 2007 to represent a time period of low solar activity and the year 2003 for high solar activity. The forthcoming analyses will already include different altitudes due to the orbital decay. The average altitudes are $493 \mathrm{~km}(2003)$ and $478 \mathrm{~km}$ (2007) for GRACE, and $408 \mathrm{~km}$ (2003) and $354 \mathrm{~km}$ (2007) for CHAMP. During 2003 and 2007 the average We further look at the sensitivity of the densities with respect to MLAT, MLT, and $F_{10.7}$ index. Here, we average over each of the three quantities separately without taking into account the other dependencies. 
The resulting variation of neutral and electron densities with MLAT, MLT, and $F_{10.7}$ index are visualised in Fig. 4 for the selected years 2003 and 2007 for both satellites. Densities are generally larger during high solar activity. The equatorial ionization anomaly (EIA, e.g., Risbeth 2000: Balan et al. 2011: Stolle et al. 2014 Emmert 2015) is clearly visible in the electron density profile with its maximum around $15^{\circ}$ MLAT in the northern hemisphere and with densities around 2-3 times larger than at the polar regions. In comparison, the neutral densities are generally large near the equator and in the northern hemisphere. An equatorial anomaly is also slightly visible in the CHAMP densities with its peak around $18^{\circ}$ MLAT. This equatorial mass density anomaly (EMA) has already been reported in Liu et al. (2007); Heelis et al. (2010). Neutral densities also peak at $75^{\circ}$ MLAT, which is known as cusp-related mass density anomaly occurring at daytime as pointed out in Lühr et al. (2004). Both densities also depend on the magnetic local time (Fig. 4. middle). The electron densities have a clear minimum around 5h MLT, which coincides with the minimum in neutral density.

In addition to the sensitivity of both densities to MLT and MLAT, neutral densities increase almost linearly with the $F_{10.7}$ index - independent from geomagnetic activity and altitude (Fig. 4 , bottom). In comparison, increasing electron densities also show linear dependencies on the $F_{10.7}$ index, however, there is a shift in the amplitude of the electron density between the years of high and low solar activity. This is related to the decreasing peak height of the electron density and the decreasing electron density magnitude due to decreased solar radiation (e.g. Liu et al.|2006).

\section{Correlation between electron and neutral density}

To quantify the representation of the ionosphere-thermosphere relation in both in-situ densities and modeled densities from TIE-GCM, our hypothesis is that we can represent the strength of the relation between neutral and electron densities empirically via the Pearson's Linear Correlation Coefficient

$$
\rho=\frac{\sum_{i=1}^{N}\left(a_{i}-\overline{a_{i}}\right)\left(b_{i}-\overline{b_{i}}\right)}{\sqrt{\sum_{i=1}^{N}\left(a_{i}-\overline{a_{i}}\right)^{2} \sum_{i=1}^{N}\left(b_{i}-\overline{b_{i}}\right)^{2}}},
$$

s where $a$ and $b$ denote the time series of electron and neutral densities along the satellite orbit, respectively, and the corresponding mean values $\bar{a}$ and $\bar{b}$. The correlation coefficient is computed for densities during one orbital revolution of the satellite and then averaged over 15 orbits $(\approx 1$ day $)$. We show only positive correlations, since negative correlations occur rarely.

We compute the correlations for both measured and modelled densities for different conditions during the years 2003 and 2007 for GRACE and CHAMP data (Figs. 5 57). Since we expect changes of the 
correlations due to day- and night-time, we distinguish between correlations of densities in sunlight and in the Earth's shadow (Figs. 557 a, c). Since the percentage of shadow regions is small, the average correlations computed for the entire data set are very similar to correlations from data, where the satellite is located in sunlight. When the satellite is fully in sunlight, no correlations are visible in shadow regions, e.g. April and May 2003 for CHAMP. Negative correlations occur in shadow regions due to the very small amount of data and are therefore not considered in further discussions. Additionally, we expect the correlations to vary with MLAT because the thermospheric and ionospheric structures themselves show a latitude dependency (Fig. 4) (e.g., Lühr et al. 2004, Stolle et al. 2006 Park et al. 2009: Liu et al.|2014;

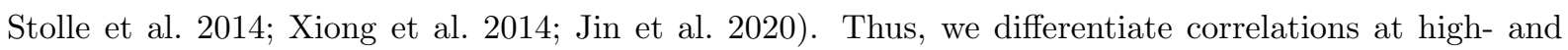
mid- and low-geomagnetic latitudes, where we define the range between $-60^{\circ}$ and $60^{\circ}$ MLAT as mid- and low-latitudes (Figs. 5017 b, d).

The most striking aspect of the computed correlations (Figs. 5.77) is the periodic behaviour. For CHAMP, the correlation reaches a minimum every 131 days, where correlations of measured data in sunlight are close to zero and correlations for modeled data drop to approximately 0.3. An exception is visible at the end of July 2007 in the CHAMP measurements, which might be related to larger data gaps at midlatitudes, since the cycles are clearly visible above and below $-60^{\circ}$ MLAT. For the GRACE mission, we do not detect the period of 131 days, instead we find a minimum close to zero in the correlations of both measured and modeled data every 160 days. The period of 131 and 160 days, respectively, correspond to the time, which is required to cover all local times at the equator considering both descending and ascending orbits, and has also been reported in Xiong et al. (2010, 2018). The MLT precession is about 11 and 13 days for $1 \mathrm{~h}$ MLT for CHAMP and GRACE, respectively. From the clearly visible periodic change in the correlations of both measured and modeled data, we conclude that the local time dependency is a large driver of changes in the correlations.

In addition, Figs. 5017 reflect that the correlation of modeled electron and neutral densities appears generally larger as compared to what we see in in-situ observations. This statement can be confirmed in the comparison of annual correlations of neutral and electron densities for modeled and measured data of both satellites in 2003 and 2007 in Tab. 1 . For example, the correlations of modeled CHAMP densities in 2003 with a mean value of 0.68 are on average 0.22 higher than the correlations of measured densities. Higher correlations of modeled data were expected, since measurements contain errors and the model is an approximation of the complex physical processes in reality, where input data might be uncertain and 
not all processes are implemented in the model (Quian et al. 2014, Emmert 2015). Weaknesses in the model, which might be related to IT-coupling, have been reported in Sutton et al. (2016), e.g., possible inaccuracy in the quadratic solver for electron density or underestimated day/night neutral temperature and density gradients in the upper thermosphere. Moreover, the correlation of modeled densities with up to 0.96 in the Earth's shadow are again much larger than those of measured data, especially shortly before the satellite is fully in sunlight again, e.g., for CHAMP in 2003 between end of August till mid-September (see Fig. 2 bottom). In this case, the shadow regions are located mainly in the southern hemisphere, and thus correlations are also large below $-60^{\circ}$ MLAT.

We find that correlations of modeled densities show generally less fluctuations than those of measured densities (see also the standard deviations in Tab. 1). This is related to the resolution of the model output, which is saved every 10 seconds and then interpolated to the 10 second resolution of the measurement, leading to a smoother time series. Generally, averaging correlations over a larger number of orbits, would simply lead to smoother results. Interestingly, the correlations of measured electron and neutral densities are highly variable at high latitudes and they show larger variations than at mid-latitudes. Reasons for this behaviour might be thermospheric winds at high latitudes (Dhadly et al. 2017), thermospheric up-welling (Lühr et al. 2004), or the generally high variability of the ionosphere in the polar regions (Liu et al. 2014, Jin et al. 2020).

Interestingly, for both missions the discrepancy between the mean correlation of measured and modeled data in 2003 with values above 0.16 is much larger than in 2007 with differences below 0.07 . We suspect that the similarity of the correlations in 2007 might either be related to changes in solar activity or to the fact that the model performance is more reliable at lower altitudes. To test the dependency of the correlations on the solar activity, we tried to conduct another experiment, where correlations are computed only from data, where the geomagnetic disturbance index $K p$ is below a certain value (e.g., 4$)$. However, the resulting correlations differ only in that they are noisier, which is probably due to a larger number of data gaps, and it does not help to further support our findings. We also looked at correlations with respect to $F_{10.7}$, however, no clear dependency of the correlations in the solar index has been found. Although our experiments did not find a clear reason for the similarity of the correlations in 2007, this does not mean that the solar activity and the model performance during low/high solar activity have no impact on changes in the correlations.

Kodikara and Zhang (2019) has shown that the assimilation of electron densities leads to improvements 
of the neutral density during low solar activity. We suspect that the reason for their success is related to the fact that their model ensemble during these periods is more skillful in representing true correlations. At this point, we take a closer look at findings from other studies to better understand the discrepancy during high solar activity. Liu et al. (2007) found that EMA is stronger at high solar activity. However, Emmert (2015) summarize that modeling variations of the equatorial anomaly has not been successful yet. This is in line with the findings by Kodikara and Zhang (2019), who conclude that further improvement is required in modeling the plasma-neutral interactions in TIE-GCM. From these studies, we suppose that the models lack in representing EMA especially during high solar activity, which might cause the discrepancy between correlations of modeled and measured data during high solar activity.

Furthermore, solar storms are visible in correlations of both modelled and measured densities. For example, during the three strongest geomagnetic storms during the year 2003 (May 29, October 2931, November 20) correlations between neutral and electron densities decrease abruptly, especially for correlations of modeled data (Fig. 5). At mid-latitudes, the correlations of modeled CHAMP data decrease by up to 0.28 during the Halloween storm (October 29-31), whereas correlations of measured data decrease by less than 0.1 . We observe that strong storms cause larger decreases in correlations of both measured and modeled data. This could be related to much faster variations in measured densities than in model predictions during storm times.

When comparing the correlations obtained for GRACE and CHAMP data, correlations of measured data agree well for both missions in 2003 (CHAMP: 0.47, GRACE: 0.48) and in 2007 (CHAMP: 0.52, GRACE: 0.53), which is not the case for modeled data. We conclude that an altitude-dependency of the correlations is not very likely because we would expect this to be visible in the results of measured and modeled data. Instead, the performance of TIE-GCM might differ with altitude and solar activity.

As discussed above, we found that the dependency of correlations between neutral and electron densities on the MLT causes a strong periodicity. Thus, we take a closer look at the correlations of densities with respect to MLT at the equator. MLT differs by 12 hours between ascending and descending nodes, 9 thus we compute correlations separately for ascending and descending parts of the orbit. In Fig. 8 , correlations are averaged over 15 ascending and descending orbits, respectively. Apparently, correlations for both modeled and measured densities vary strongly with MLT. The correlations increase around 6-9h 352 MLT after leaving the shadow regions and decrease again after 20h MLT when entering the shadow. This 53 holds for both years and thus different solar activity levels, and also for both satellite missions, e.g., at 
Table 1. Statistics of correlations between neutral and electron densities.

\begin{tabular}{lcccc}
\hline & \multicolumn{2}{c}{ CHAMP } & \multicolumn{2}{c}{ GRACE } \\
& modeled & measured & modeled & measured \\
\hline 2003 max & 0.92 & 0.75 & 0.91 & 0.76 \\
2003 mean & 0.68 & 0.47 & 0.64 & 0.48 \\
2003 std & 0.17 & 0.20 & 0.21 & 0.22 \\
\hline 2007 max & 0.81 & 0.88 & 0.92 & 0.84 \\
2007 mean & 0.58 & 0.52 & 0.60 & 0.53 \\
2007 std & 0.14 & 0.18 & 0.19 & 0.23 \\
\hline
\end{tabular}

different altitudes. Between 12 and 18h MLT, correlations are generally large with values above 0.5 and correlations of modeled densities are larger than those from measured densities. Interestingly, there are clear differences between correlations of ascending and descending orbits, especially for modeled data at daytime. This might be related to a seasonal dependency of the correlations, since correlations are larger during winter on the northern hemisphere. We can only speculate that the seasonal signal in modeled densities is overestimated, since this is not seen in the measurements. Around 6h MLT, correlations of measured densities peak with values around 0.8. On the contrary, correlations of modeled densities are generally small at 6h MLT except for GRACE data in 2003, where correlations of modeled and measured data agree well. Instead, correlations of modeled densities peak after noon between 12 and $18 \mathrm{~h}$ MLT, which changes with solar activity and season.

We suppose that the strong MLT-dependency of the correlation is related to the peak in neutral and electron density following the sub-solar point. Also, the latitude variations of the EMA in rough follows those of the EIA (Liu et al. 2007). However, the EMA is not visible after midnight, which is not the case for EIA. Another reason might be the occurrence of neutral density depletions and plasma bubbles around 21h MLT (Stolle et al. 2006 , Park et al. 2009, Xiong et al. 2010, Park et al. 2010$)$.

From the above analyses, we are now aware of several conditions, where in-situ data and model simulations agree well. We found that correlations of both measured and modeled densities increase around 6-9h MLT after leaving the shadow regions and decrease again after 20h MLT when entering the shadow. In addition, correlations are generally high $(>0.5)$ during daytime with its largest values between 12 and $18 \mathrm{~h}$ MLT, which changes with solar activity and season. In addition, we note that the model generally over- 
estimates correlations as compared to what we observe. Especially during high solar activity, the model overestimates the correlations by about 0.2 on average, while under low solar activity the differences are below 0.1 , which might again be related to faster and stronger variations in measured densities than in model predictions during high solar activity. Here, we expect that assimilating electron densities would improve the model performance less than during low solar activity, since high correlations are important for the success of the data assimilation. During storm times, which are known as special conditions, we note that the model strongly overestimates correlations compared to what we observe. Nevertheless, we expect that the assimilation of measured densities will reduce this overestimation.

\section{Conclusions}

To study the relation between neutral and electron densities in models and measurements, we propose that the strength of this relation can be represented empirically via the correlation coefficient. In this study, we extensively compare correlations between electron and neutral densities from direct in-situ observations with correlations from densities of the physical model TIE-GCM, to see, if the model represents the observed correlations well. We argue that if the model correlations correspond well to what is observed, predicting neutral density by assimilating ionospheric observations is most promising.

We obtain in-situ neutral densities from accelerometer measurements on-board the satellite missions GRACE and CHAMP. We use CHAMP electron densities from the PLP instrument and GRACE electron densities derived from K-Band measurements. Here, we focus on the years 2003 and 2007 representing conditions of high and low solar activity, respectively.

Our results show that correlations of electron and neutral densities strongly depend on MLT with a correlation at 131 day periodicity for CHAMP and 160 day periodicity for GRACE, which corresponds to the orbital local time precession of the missions. Correlations increase around 6-9h MLT after leaving the shadow regions. During daytime, correlations are generally high $(>0.5)$ and decrease again after 20h MLT when entering the shadow. For measured data, the correlations peak around $6 \mathrm{~h}$ MLT. On the contrary, we note that the correlation in the model is generally overestimated, especially during high solar activity, where the differences between correlations of modeled and measured densities is about 0.2 . We also have more trust in the model during low solar activity, since the correlations agree well for both satellites. During storm times, which are known as special conditions, correlations decrease abruptly, especially for modeled densities where correlations drop by up to 0.7 . 

${ }_{411}$ run in the future.

\section{${ }_{412}$ Declarations}

422 None of the authors have competing interest.

\section{$423 \quad$ Funding}

424 The authors are grateful to the research grant through the TIPOD project (FKZ.: KU 1207/27-1) 425 supported by the German Research Foundation under SPP 1788 (DynamicEarth) and the TIK Project ${ }_{426}$ (FKZ.: 50 LZ 1606) supported by the German Aerospace Center (DLR). This work is partly supported ${ }_{427}$ by the TIRO project under subcontract No. SW-CO-DTUGS-126 within ESA's DISC under contract ${ }_{428}$ No. $4000109587 / 13 / \mathrm{I}-\mathrm{NB}$. 

434 and approved the final manuscript.

\section{Authors' contributions}

\section{Acknowledgments}

Not applicable.

\section{References} doi:10.1007/s00190-011-0449-z 116(A7). doi:10.1029/2010JA016399

KV computed the neutral densities, conducted the research, and wrote large parts of the manuscript. AC was responsible for the TIE-GCM runs and the wavelet analyses. CX and CS computed GRACE electron density data and contributed helpful suggestions on the development of the results. JK helped to refine the draft and contributed to the writing. All authors helped to revise the manuscript. All authors read

Alizadeh, MM, H Schuh, S Todorova, and M Schmidt (2011) Global ionosphere maps of VTEC from GNSS, satellite altimetry, and Formosat-3/COSMIC data. Journal of Geodesy 85(12):975-987.

Anderson, J, T Hoar, K Raeder, H Liu, N Collins, R Torn, and A Avellano (2009) The data assimilation research testbed: a community facility. Bulletin of the American Meteorological Society, 90(9):1283-1296. doi:10.1175/2009BAMS2618.1

Balan, N, M Yamamoto, JY Liu, Y Otsuka, H Liu, and H Lühr (2011) New aspects of thermospheric and ionospheric storms revealed by CHAMP. Journal of Geophysical Research: Space Physics,

Bettadpur, S (2012) Gravity recovery and climate experiment: Product specification document. Tech. Rep. GRACE 327-720. Center for Space Research, The University of Texas at Austin

Bowman, B (2002) True satellite ballistic coefficient determination for HASDM. In AIAA/AAS Astrodynamics Specialist Conference and Exhibit. Monterey, California: American Institute of Aeronautics and Astronautics. doi:10.2514/6.2002-4887

Bowman, B, WK Tobiska, F Marcos, C Huang, C Lin, and W Burke (2008) A new empirical thermospheric density model JB2008 using new solar and geomagnetic indices. In AIAA/AAS Astrodynamics specialist conference and exhibit, pp.6438, doi:10.2514/6.2008-6438.

Bruinsma, S (2021) DTM2020. Mini-ISWAT Virtual Meeting Series: G2A Atmosphere Variability, 22-26 February 2021, https://www.iswat-cospar.org/virtual-meeting-series_G2A. 
Carrere, L, F Lyard, M Cancet, and A Guillot (2015) FES 2014, a new tidal model on the global ocean with enhanced accuracy in shallow seas and in the arctic region. In Geophysical Research Abstracts (Vol. 17, p. 5481). Vienna, Austria.

Case, K, G Kruizinga and SC Wu (2010) GRACE Level 1B data product user handbook. Tech. Rep. JPL D-22027. Jet Propulsion Laboratory

CERES Science Team (2019) NASA Atmospheric Science Data Center (ASDC), Accessed SYN1deg-1Hour at doi:10.5067/Terra+Aqua/CERES/SYN1deg1Hour_L3.004

Codrescu, MV, C Negrea, M Fedrizzi, TJ Fuller-Rowell, A Dobin, N Jakowsky, H Khalsa, T Matsuo and N Maruyama (2014) A real-time run of the Coupled Thermosphere Ionosphere Plasmasphere Electrodynamics (CTIPe) model. Space Weather, 10(2). doi:10.1029/2011SW000736

Dettmering, D, M Limberger and M Schmidt (2014) Using DORIS measurements for modeling the vertical total electron content of the Earth's ionosphere. Journal of Geodesy, 88(12):1131-1143. doi:10.1007/s00190-014-0748-2

Dhadly, M, J Emmert, D Drob, M Conde, E Doornbos, G Shepherd, J Makela, Q Wu, R Niciejewski, and A Ridley (2017) Seasonal dependence of northern high-latitude upper thermospheric winds: A quiet time climatological study based on ground-based and space-based measurements: Seasonal dependence on neutral winds. Journal of Geophysical Research: Space Physics, 122(2):2619-2644. doi:10.1002/2016JA023688

Dobslaw, H, I Bergmann-Wolf, R Dill, L Poropat, M Thomas, C Dahle, S Esselborn, R König and F Flechtner(2017) A new high-resolution model of non-tidal atmosphere and ocean mass variability for de-aliasing of satellite gravity observations: AOD1B RL06. Geophysical Journal International, 211(1):263-269. doi:10.1093/gji/ggx302

Doornbos, E (2012). Thermospheric density and wind determination from satellite dynamics. Springer Science \& Business Media, Berlin. doi:10.1007/978-3-642-25129-0.

Doornbos, E, M Forster, B Fritsche, T van Helleputte, J van den IJssel, G Koppenwallner, H Lühr, D Rees and P Visser (2009) ESTEC Contract 21022/07/NL/HE Air density models derived from multi-satellite drag observations - final report. DEOS/Delft Univ. of Technology Scientific TR, 1 , $7-39$.

Emmert, JT (2015) Thermospheric mass density: A review. Advances in Space Research, 56(5):773-824. doi: 10.1016/j.asr.2015.05.038 
Emmert, JT, DP Drob, JM Picone, DE Siskind, M Jones, MG Mlynczak, PF Bernath, X Chu, E Doornbos, B Funke, LP Goncharenko, ME Hervig, MJ Schwartz, PE Sheese, F Vargas, BP Williams and T Yuan (2020) NRLMSIS 2.0: A whole-atmosphere empirical model of temperature and neutral species densities. Earth and Space Science, 7, e2020EA001321. doi:10.1029/2020EA001321

Evensen, G (2009) Data Assimilation. The Ensemble Kalman Filter. (Second ed.). Springer Berlin Heidelberg, Germany.

Goss, A, M Schmidt, E Erdogan, B Görres and F Seitz (2019) High-resolution vertical total electron content maps based on multi-scale B-Spline representations. Annales Geophysicae, 37 (4), 699-717. doi: 10.5194/angeo-37-699-2019

Hagan, ME, A Maute and RG Roble (2009) Tropospheric tidal effects on the middle and upper atmosphere. Journal of Geophysical Research: Space Physics, 114 (A1). doi:10.1029/2008JA013637

Hajj, GA, BD Wilson, C Wang, X Pi and IG Rosen (2004) Data assimilation of ground GPS total electron content into a physics-based ionospheric model by use of the Kalman filter. Radio Science, 39(1). doi:10.1029/2002RS002859

Häusler, K and H Lühr (2009) Nonmigrating tidal signals in the upper thermospheric zonal wind at equatorial latitudes as observed by CHAMP. Annales Geophysicae, 27(7):2643-2652. doi:10.5194/angeo-27-2643-2009

Heelis, RA, JK Lowell and RW Spiro (1982) A model of the high-latitude ionospheric convection pattern. Journal of Geophysical Research: Space Physics, 87(A8):6339-6345. doi:10.1029/JA087iA08p06339

Hernández-Pajares M, D Roma-Dollase, A Krankowski, A García-Rigo, and R Orús-Pérez (2017) Methodology and consistency of slant and vertical assessments for ionospheric electron content models. Journal of Geodesy, 91(12):1405-1414. doi:10.1007/s00190-017-1032-z

Immel, TJ, E Sagawa, SL England, SB Henderson, ME Hagan, SB Mende, HU Frey, CM Swenson and LJ Paxton (2006) Control of equatorial ionospheric morphology by atmospheric tides. Geophysical Research Letters, 33(15). doi:10.1029/2006GL026161

Jin, Y, C Xiong, L Clausen, A Spicher, D Kotova, S Brask, G Kervalishvili, C Stolle and W Miloch (2020) Ionospheric plasma irregularities based on in situ measurements from the Swarm satellites. Journal of Geophysical Research: Space Physics, 125(7), e2020JA028103. doi:10.1029/2020JA028103

Knocke, P, J Ries and B Tapley (1988) Earth radiation pressure effects on satellites. In: Proceedings of 
Kodikara, T and K Zhang (2019) Forecasting of the thermosphere via assimilation of electron density and temperature data. Earth and Space Science Open Archive, 30. doi:10.1002/essoar.10500883.1

Kvas, A, S Behzadpour, M Ellmer, B Klinger, S Strasser, N Zehentner and T Mayer-Gürr (2018) ITSG-Grace2018: Overview and Evaluation of a New GRACE-Only Gravity Field Time Series. Journal of Geophysical Research:Solid Earth, 124(8):9332-9344. doi:10.1029/2019JB017415

Kvas, A, T Mayer-Gürr, S Krauss, JM Brockmann, T Schubert, W-D Schuh, R Pail, T Gruber, A Jäggi and U Meyer (2019) The satellite-only gravity field model GOCO06s. GFZ Data Services. doi:10.5880/ICGEM.2019.002

Laundal, KM and AD Richmond (2017) Magnetic coordinate systems. Space Science Reviews, 206(1-4):27-59. doi:10.1007/s11214-016-0275-y

Lee, I-T, T Matsuo, AD Richmond, JY Liu, W Wang, CH Lin, JL Anderson and MQ Chen (2012) Assimilation of FORMOSAT-3/COSMIC electron density profiles into a coupled thermosphere/ionosphere model using ensemble Kalman filtering. Journal of Geophysical Research: Space Physics, 117(A10318). doi:10.1029/2012JA017700

Lei, J, JP Thayer and JM Forbes (2010) Longitudinal and geomagnetic activity modulation of the equatorial thermosphere anomaly. Journal of Geophysical Research: Space Physics, 115(A8). doi:10.1029/2009JA015177

Liu, H, H Lühr and S Watanabe (2007) Climatology of the equatorial thermospheric mass density anomaly. Journal of Geophysical Research: Space Physics, 112(A5). doi:10.1029/2006JA012199

Liu, J, R Chen, J An, Z Wang and J Hyyppa (2014) Spherical cap harmonic analysis of the Arctic ionospheric TEC for one solar cycle. Journal of Geophysical Research: Space Physics, 119(1):601-619. doi:10.1002/2013JA019501

Liu, L, W Wan, B Ning, OM Pirog and VI Kurkin (2006) Solar activity variations of the ionospheric peak electron density. Journal of Geophysical Research: Space Physics, 111(A8). doi:10.1029/2006JA011598

Lühr, H, M Rother, W Köhler, P Ritter and L Grunwaldt (2004) Thermospheric up-welling in the cusp region: Evidence from CHAMP observations. Geophysical Research Letters, 31(6). doi:10.1029/2003GL019314

Matsuo, T, I-T Lee and JL Anderson (2013) Thermospheric mass density specification using an 
ensemble Kalman filter. Journal of Geophysical Research: Space Physics, 118(3):1339-1350. doi: $10.1002 /$ jgra. 50162

McNamara, LF, DL Cooke, CE Valladares and BW Reinisch (2007) Comparison of CHAMP and Digisonde plasma frequencies at Jicamarca, Peru. Radio Science, 42(2). doi:10.1029/2006RS003491

Nerger, L and W Hiller (2013) Software for ensemble-based data assimilation systems-Implementation strategies and scalability. Computers \& Geosciences, 55, 110-118. doi:10.1016/j.cageo.2012.03.026

Oberheide, J, Q Wu, TL Killeen, ME Hagan and RG Roble (2006) Diurnal nonmigrating tides from TIMED Doppler Interferometer wind data: Monthly climatologies and seasonal variations. Journal of Geophysical Research: Space Physics, 111(A10). doi:10.1029/2005JA011491

Palmroth, M, M Grandin, T Sarris, E Doornbos, S Tourgaidis, A Aikio, S Buchert, MA Clilverd, I Dandouras, R Heelis, A Hoffmann, N Ivchenko, G Kervalishvili, DJ Knudsen, A Kotova, H-L Liu, DM Malaspina, G March, A Marchaudon, O Marghitu, T Matsuo, WJ Miloch, T Moretto-Jørgensen, D Mpaloukidis, N Olsen, K Papadakis, R Pfaff, P Pirnaris, C Siemes, C Stolle, J Suni, J van den IJssel, PT Verronen, P Visser, M Yamauchi (2021) Lower-thermosphere-ionosphere (LTI) quantities: current status of measuring techniques and models. Annales Geophysicae, 39:189-237, doi:10.5194/angeo-39-189-2021

Pardini, C, L Anselmo, K Moe and MM Moe (2010) Drag and energy accommodation coefficients during sunspot maximum. Advances in Space Research, 45(5):638-650. doi:10.1016/j.asr.2009.08.034

Park, J, H Lühr and KW Min (2010) Neutral density depletions associated with equatorial plasma bubbles as observed by the CHAMP satellite. Journal of Atmospheric and Solar-Terrestrial Physics, 72(2):157-163. doi:10.1016/j.jastp.2009.11.003

Park, J, H Lühr, C Stolle, M Rother, KW Min and I Michaelis (2009) The characteristics of field-aligned currents associated with equatorial plasma bubbles as observed by the CHAMP satellite. Annales Geophysicae, 27(7):2685-2697. doi:10.5194/angeo-27-2685-2009

Prange, L (2010) Global gravity field determination using the GPS measurements made onboard the low earth orbiting satellite CHAMP. Dissertation, Institut für Geodäsie und Photogrammetrie, Geodätisch-geophysikalische Arbeiten in der Schweiz Vol. 81

Prange, L, A Jäggi, R Dach, H Bock, G Beutler and L Mervart (2010) AIUB-CHAMP02S: The influence of GNSS model changes on gravity field recovery using spaceborne GPS. Advances in Space Research, 45(2):215-224. doi:10.1016/j.asr.2009.09.020 
Qian, L, AG Burns, BA Emery, B Foster, G Lu, A Maute, AD Richmond, RG Roble, SC Solomon and W Wang (2014) The NCAR TIE-GCM: A community model of the coupled thermosphere/ionosphere system. Modeling the Ionosphere-Thermosphere System, 201 ,73-83.

Reigber, C, H Lühr and P Schwintzer (2002). Champ mission status. Advances in Space Research, 30(2):129-134, doi:10.1016/S0273-1177(02)00276-4.

Risbeth H (2000) The equatorial F-layer: Progress and puzzles. Annales Geophysicae, 18, 730-739. doi: $10.1007 / \mathrm{s} 00585-000-0730-6$

Sarris, TE, ER Talaat, M Palmroth, I Dandouras, E Armandillo, G Kervalishvili, S Buchert, S Tourgaidis, DM Malaspina, AN Jaynes, N Paschalidis, J Sample, J Halekas, E Doornbos, V Lappas, T Moretto Jørgensen, C Stolle, M Clilverd, Q Wu, I Sandberg, P Pirnaris and A Aikio (2020)

Daedalus: A low-flying spacecraft for in situ exploration of the lower thermosphere-ionosphere. Geoscientific Instrumentation, Methods and Data Systems, 9(1):153-191. doi:10.5194/gi-9-153-2020

Schunk, RW, L Gardner, L Scherliess and L Zhu (2012) Problems associated with uncertain parameters and missing physics for long-term ionosphere-thermosphere forecasting:

IONOSPHERE-THERMOSPHERE FORECASTING. Radio Science, 47(4). doi: 10.1029/2011RS004911

Siddiqui, T, Y Yamazaki, C Stolle, H Lühr, J Matzka, A Maute and N Pedatella (2018) Dependence of lunar tide of the equatorial electrojet on the winter-time polar vortex, solar flux and QBO.

Geophysical Research Letters, 45(9):3801-3810. doi:10.1029/2018GL077510

Stolle, C and H Liu (2014) Low-Latitude Ionosphere and Thermosphere. In Modeling the Ionosphere-Thermosphere System (pp. 259-272). American Geophysical Union (AGU). doi:10.1002/9781118704417.ch21

Stolle, C, H Lühr, M Rother and G Balasis (2006) Magnetic signatures of equatorial spread F as observed by the CHAMP satellite. Journal of Geophysical Research: Space Physics, 111(A2). doi:10.1029/2005JA011184

Suttles, JT, RN Green, P Minnis, GL Smith, WF Staylor, BA Wielicki, IJ Walker, DF Young, VR Taylor and LL Stowe (1988) Angular radiation models for Earth-atmosphere system: Volume I shortwave radiation. Reference Publication No. NASA RP-1184. Hampton, VA: NASA Langley Research Center.

Suttles, JT, RN Green, P Minnis, GL Smith, WF Staylor, BA Wielicki, IJ Walker, DF Young, VR 
Taylor and LL Stowe (1989) Angular radiation models for Earth-atmosphere system: Volume II longwave radiation. Reference Publication No. NASA RP-1184. Hampton, VA: NASA Langley Research Center.

Sutton, EK, S Solomon, AG Burns, BA Emery, B Foster, H Liu, G Lu, A Maute, J McInerney, NM Pedatella, L Qian, A Richmond, R Roble, W Wang and Q Wu (2016) TIE-GCM v. 2.0. Santa Fe, New Mexico.

Tapley, BD, S Bettadpur, M Watkins and C Reigber (2004) The gravity recovery and climate experiment: Mission overview and early results. Geophysical Research Letters, 31(9), doi:10.1029/2004GL019920.

Vielberg, K, E Forootan, C Lück, A Löcher, J Kusche and K Börger (2018) Comparison of accelerometer data calibration methods used in thermospheric neutral density estimation. Annales Geophysicae, 36(3):761-779.doi:10.5194/angeo-36-761-2018

Vielberg, K and J Kusche (2020) Extended forward and inverse modeling of radiation pressure accelerations for LEO satellites. Journal of Geodesy, 94(4):43. doi:10.1007/s00190-020-01368-6

Weimer, DR (2005) Predicting surface geomagnetic variations using ionospheric electrodynamic models. Journal of Geophysical Research: Space Physics, 110(A12). doi:10.1029/2005JA011270

Xiong, C, H Lühr, SY Ma and K Schlegel (2015) Validation of GRACE electron densities by Incoherent scatter radar data and estimation of plasma scale height in the topside ionosphere. Advances in Space Research, 55(8):2048-2057. doi:10.1016/j.asr.2014.07.022

Xiong, C, H Lühr, M Schmidt, M Bloßfeld, and S Rudenko (2018) An empirical model of the thermospheric mass density derived from CHAMP satellite. Annales Geophysicae, 36(4):1141-1152. doi:10.5194/angeo-36-1141-2018

Xiong, C, H Lühr and C Stolle (2014) Seasonal and latitudinal variations of the electron density nonmigrating tidal spectrum in the topside ionospheric $\mathrm{F}$ region as resolved from CHAMP observations. Journal of Geophysical Research:Space Physics, 119(12):10,416-10,425. doi:10.1002/2014JA020354

Xiong, C, H Lühr and C Stolle (2021) GRACE electron density derived from the K-Band Ranging System (KBR). V. 0101. GFZ Data Services. doi:10.5880/GFZ.2.3.2021.003

Xiong, C, J Park, H Lühr, C Stolle and SY Ma (2010) Comparing plasma bubble occurrence rates at CHAMP and GRACE altitudes during high and low solar activity. Annales Geophysicae, 28 (9), 

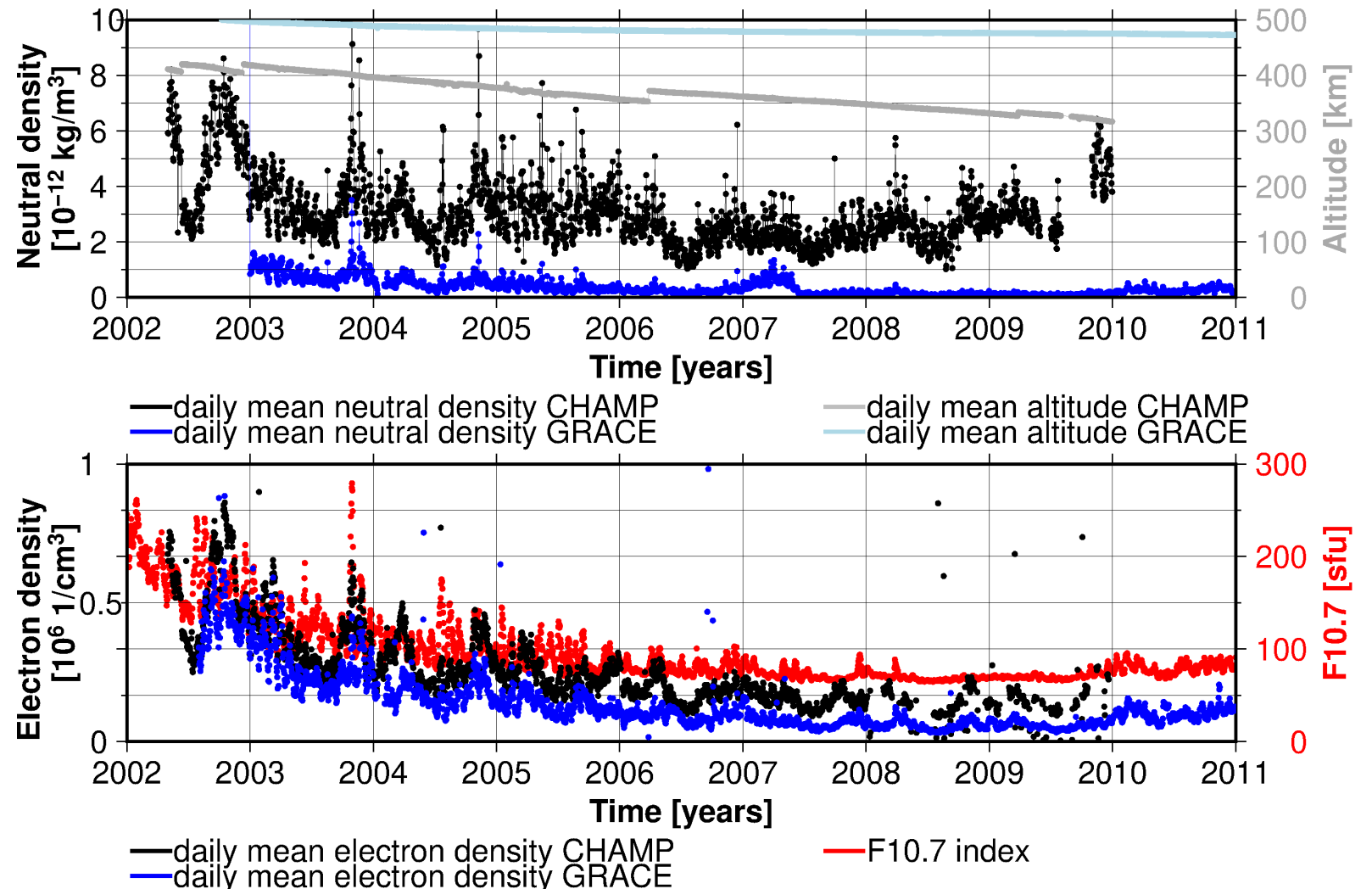

Figure 1. Daily mean neutral (top) and electron (bottom) density of the satellites CHAMP (black) and GRACE (blue) between 2002 and 2010, together with the $F_{10.7}$ index (red) and daily mean altitudes (light colors). 

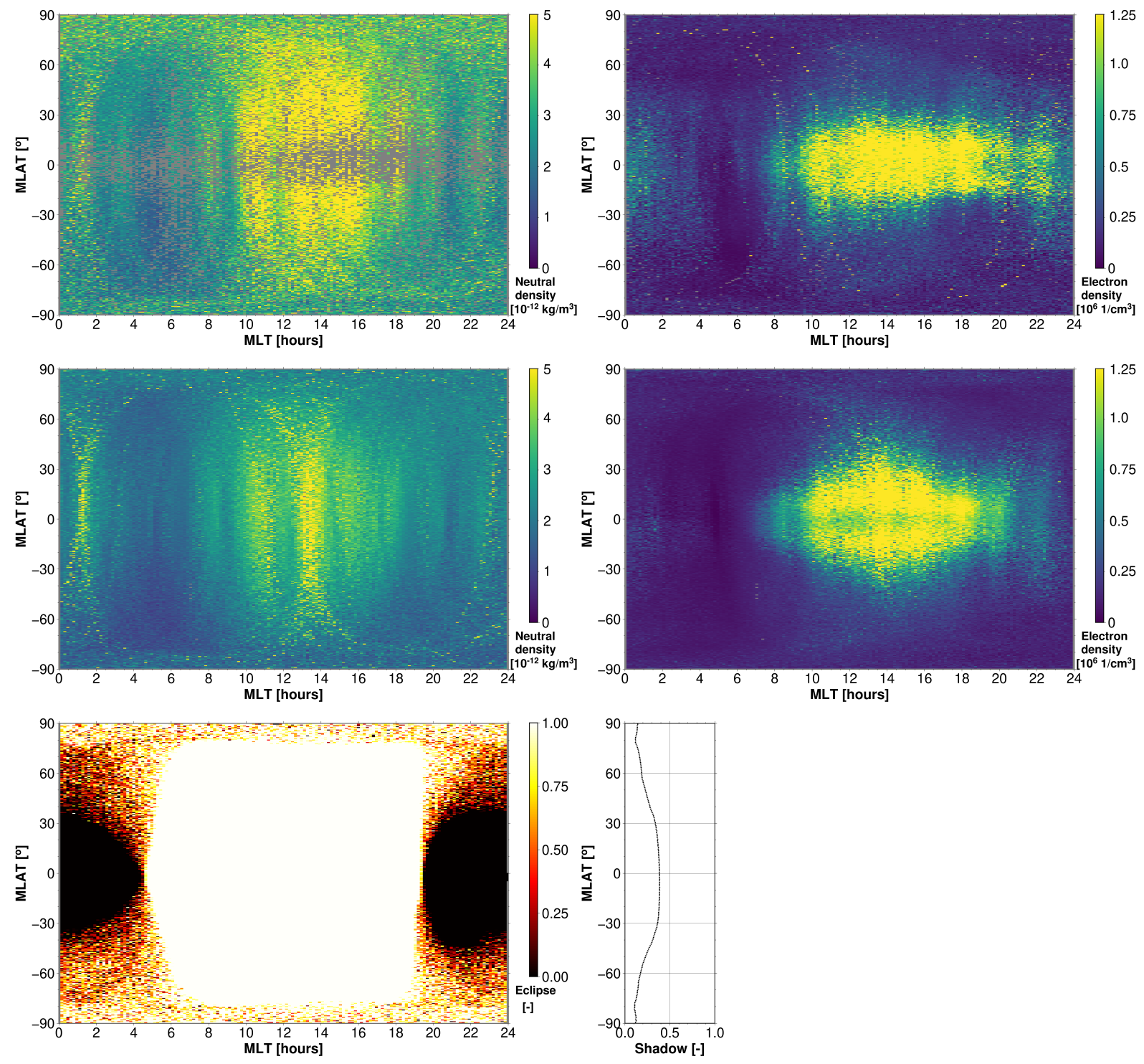

Figure 2. CHAMP neutral density (left) and electron density (right) for the whole year 2003. Data from measurements (top), modeled from TIE-GCM (middle). Note for neutral densities: gaps below 200 seconds are interpolated in all data sets. Remaining gaps (grey) in the measured neutral densities (top, left) are due to missing measurements. Bottom: Shadow function along the orbit of the CHAMP satellite for the whole year 2003 and the amount of shadow per MLAT. 


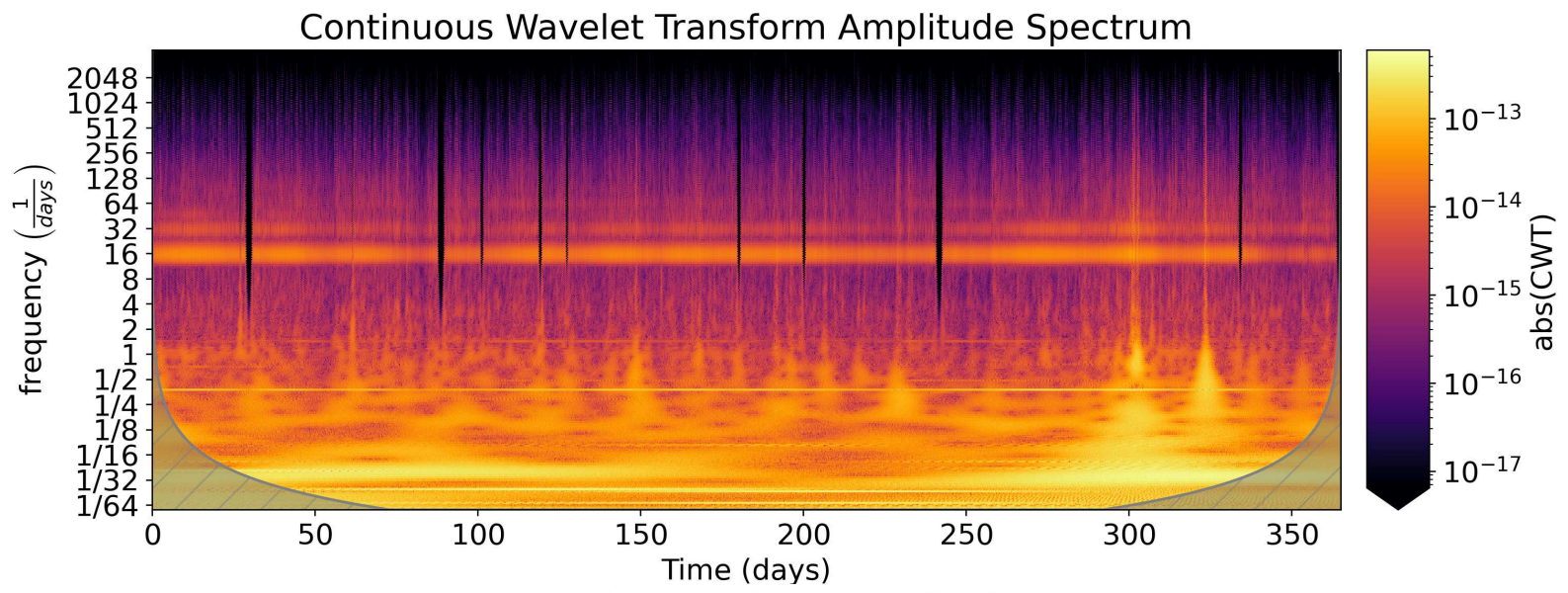

Continuous Wavelet Transform Amplitude Spectrum

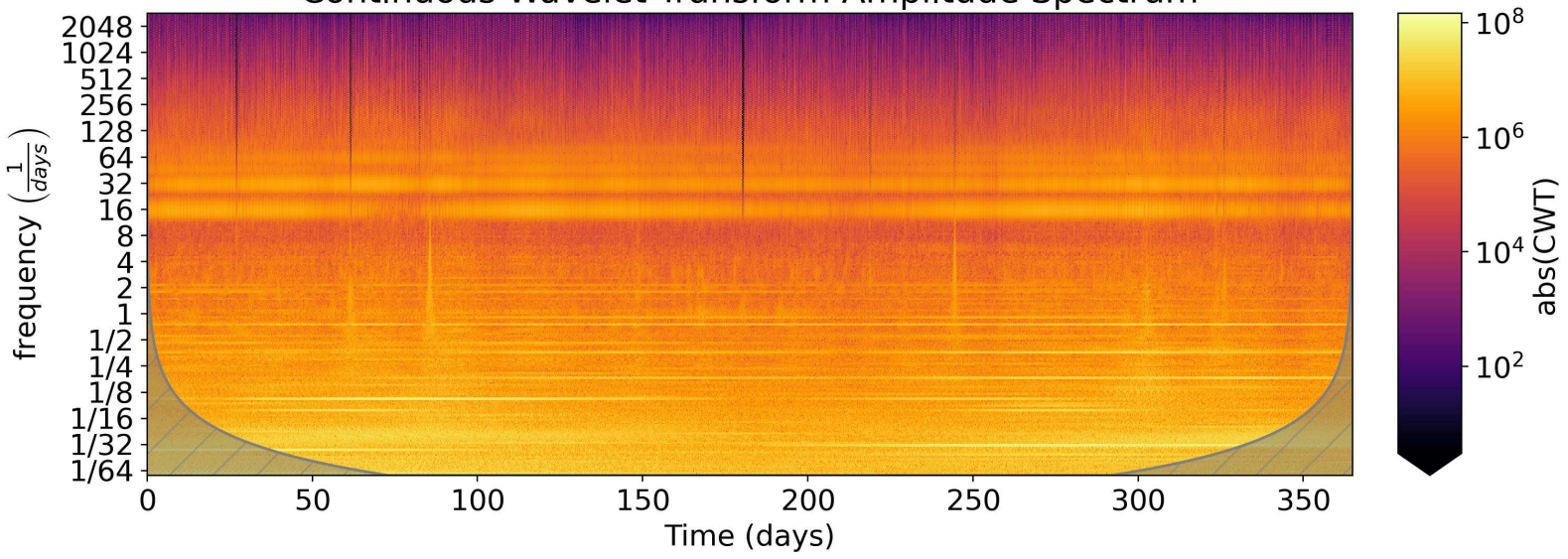

Figure 3. Wavelet analysis of CHAMP accelerometer-derived neutral densities (top) and electron densities from PLP data (bottom) for the whole year 2003. 

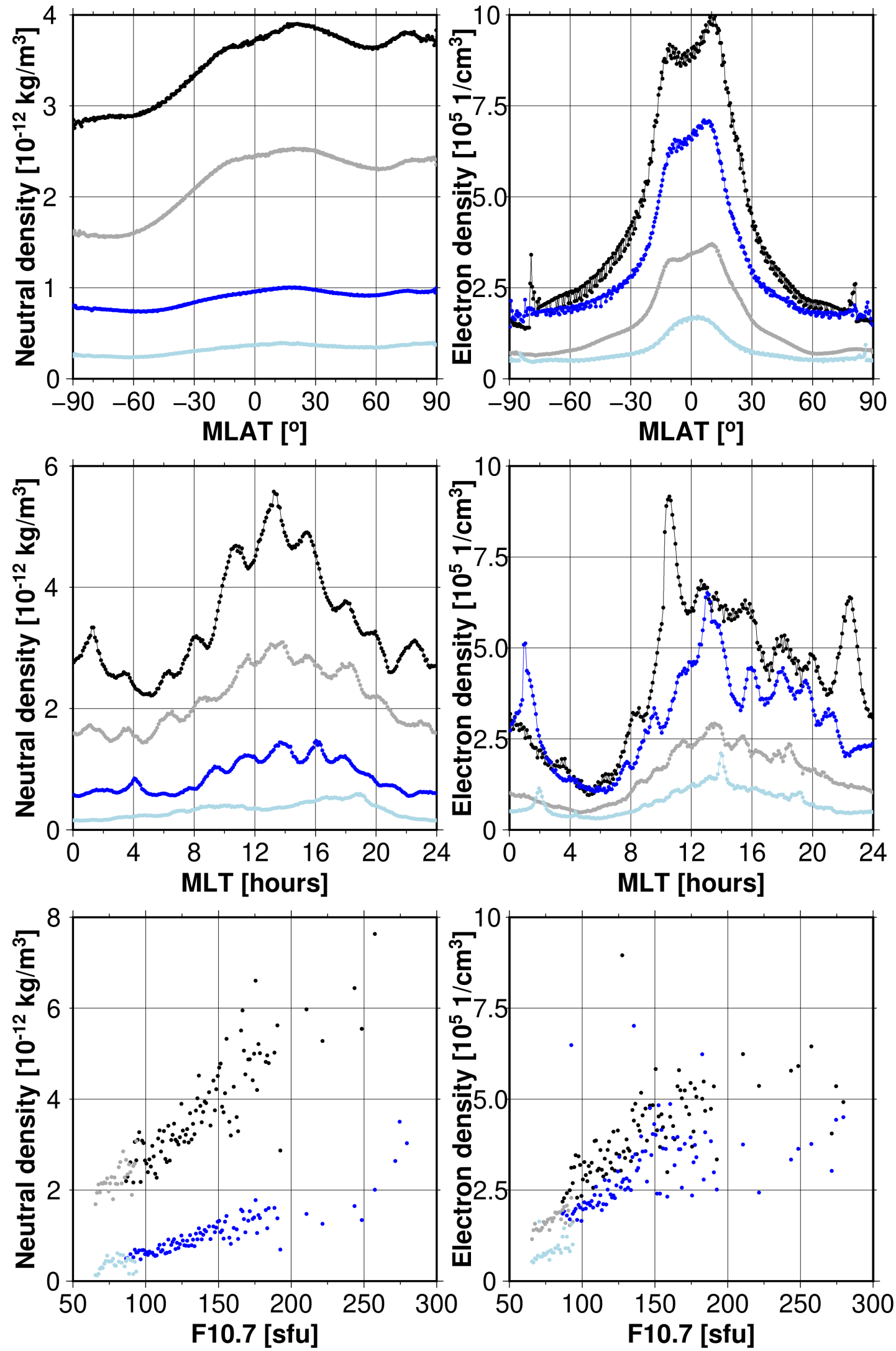

-CHAMP 2003 -CHAMP 2007 —GRACE 2003 -GRACE 2007

Figure 4. Dependencies of neutral densities (left) and electron densities (right) averaged per magnetic latitude (top), magnetic local time (middle) and daily $F_{10.7}$ index (bottom). 

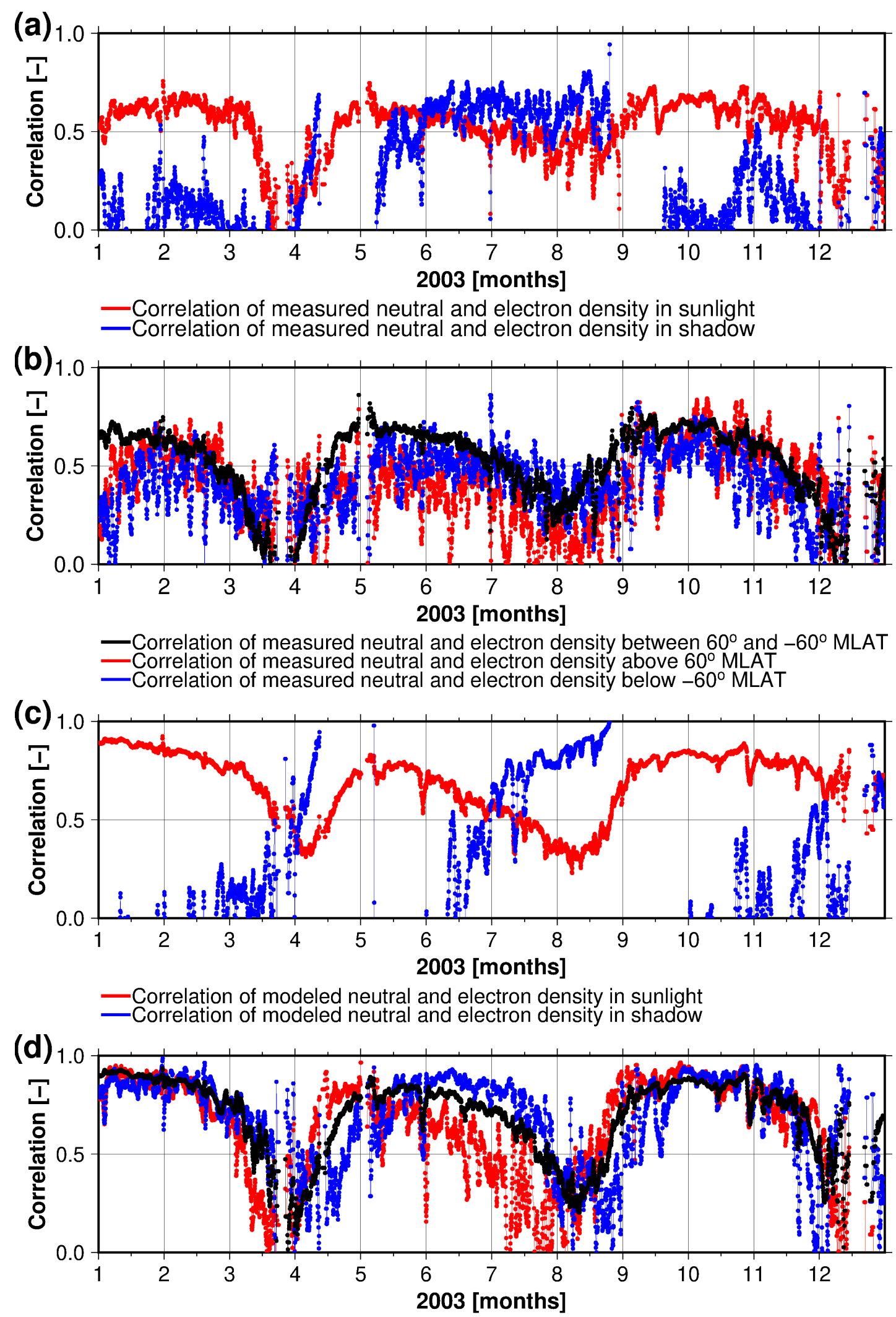

- Correlation of modeled neutral and electron density between $60^{\circ}$ and $-60^{\circ}$ MLAT - Correlation of modeled neutral and electron density above $60^{\circ} \mathrm{MLAT}$ Correlation of modeled neutral and electron densitv below $-60^{\circ} \mathrm{MLAT}$

Figure 5. Correlation analysis of measured (a, b) and modeled (c, d) CHAMP neutral and electron densities for the whole year 2003. a, c: The correlation coefficients have been computed from the available densities in sunlight or shadow per orbit, then the correlations from 15 orbits were averaged. 28

b, d: Same, but with data only at specific magnetic latitudes. 


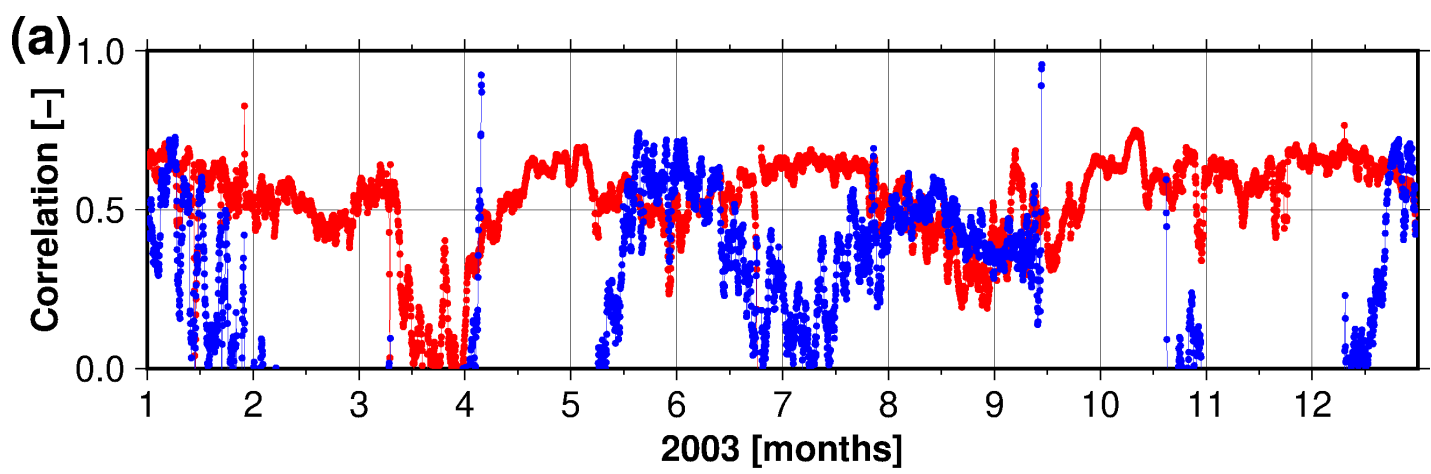

-Correlation of measured neutral and electron density in sunlight
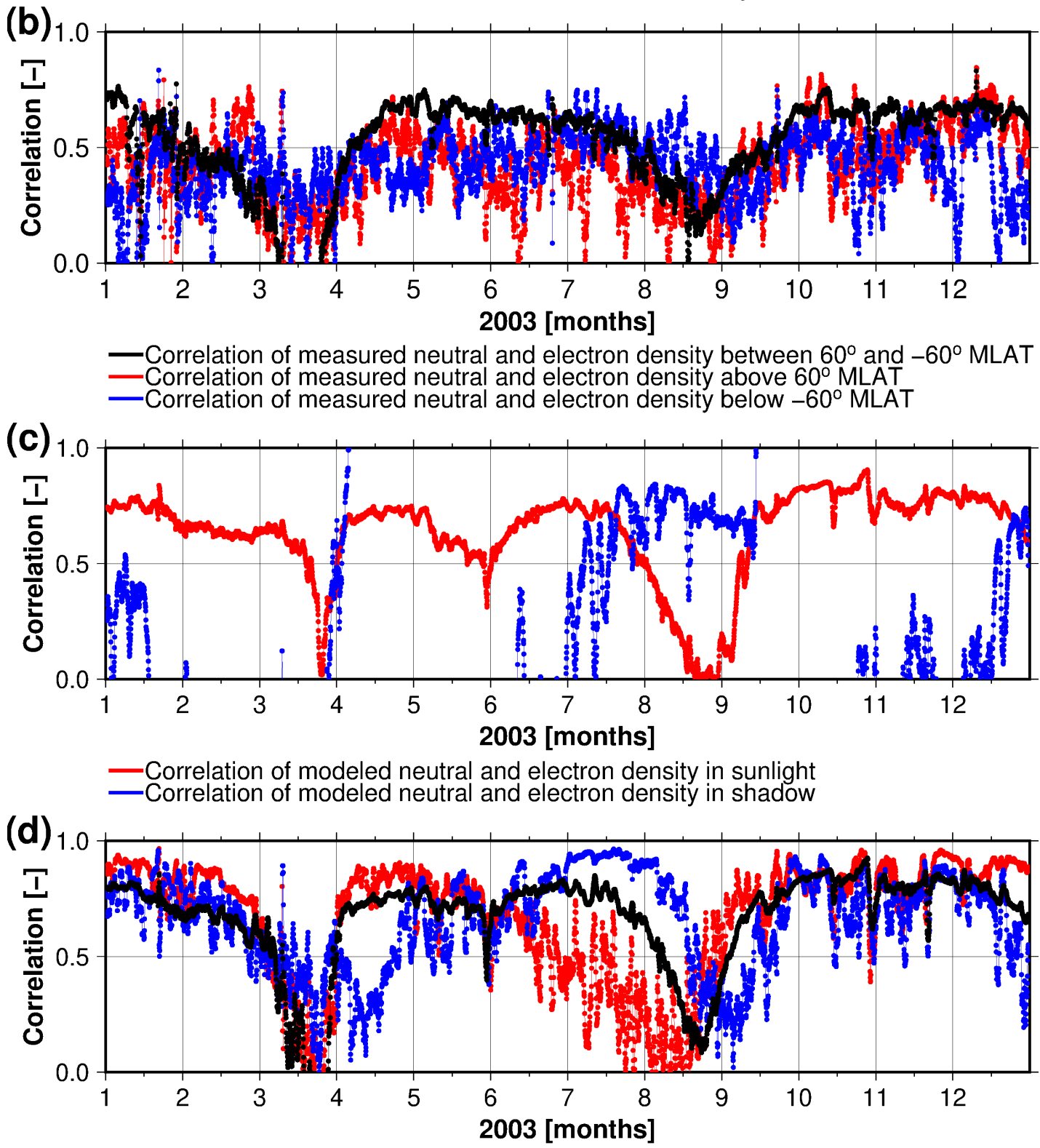

- Correlation of modeled neutral and electron density between $60^{\circ}$ and $-60^{\circ} \mathrm{MLAT}$ - Correlation of modeled neutral and electron density above $60^{\circ} \mathrm{MLAT}$ Correlation of modeled neutral and electron densitv below $-60^{\circ} \mathrm{MLAT}$

Figure 6. Correlation analysis of measured (a, b) and modeled (c, d) GRACE neutral and electron densities for the whole year 2003. a, c: The correlation coefficients have been computed from the available densities in sunlight or shadow per orbit, then the correlations from 15 orbits were averaged. 

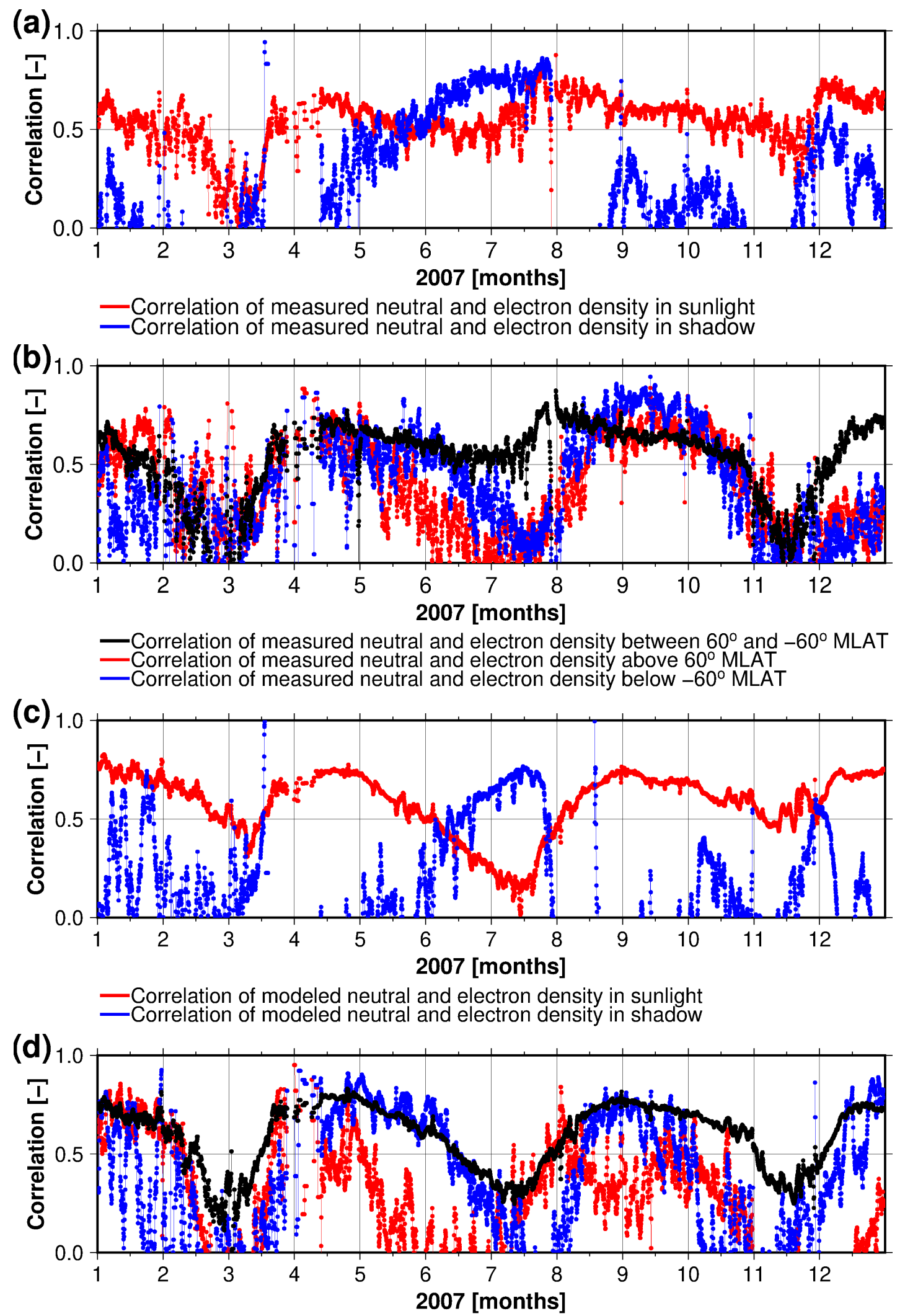

- Correlation of modeled neutral and electron density between $60^{\circ}$ and $-60^{\circ}$ MLAT - Correlation of modeled neutral and electron density above $60^{\circ} \mathrm{MLAT}$ Correlation of modeled neutral and electron densitv below $-60^{\circ} \mathrm{MLAT}$

Figure 7. Correlation analysis of measured (a, b) and modeled (c, d) CHAMP neutral and electron densities for the whole year 2007. a, c: The correlation coefficients have been computed from the available densities in sunlight or shadow per orbit, then the correlations from 15 orbits were averaged. 

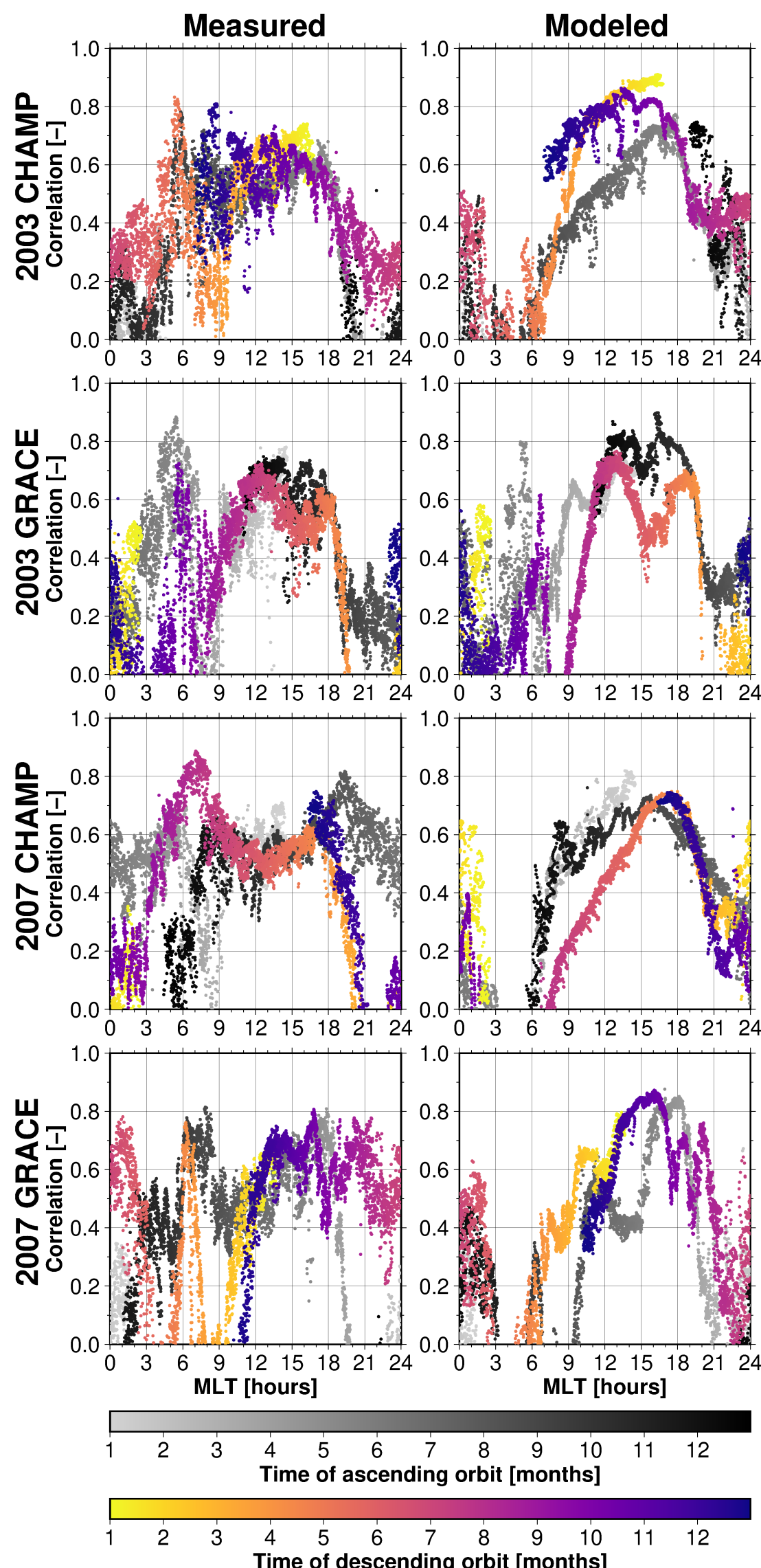

Figure 8. Correlation coefficients of neutral and electron densities with respect to magnetic latitude for measured data (left column) and modeled data (right column) for GRACE and CHAMP during the years 2003 and 2007. The correlation coefficients have been averaged over 15 ascending and 15 descending orbits, respectively. 
Figures
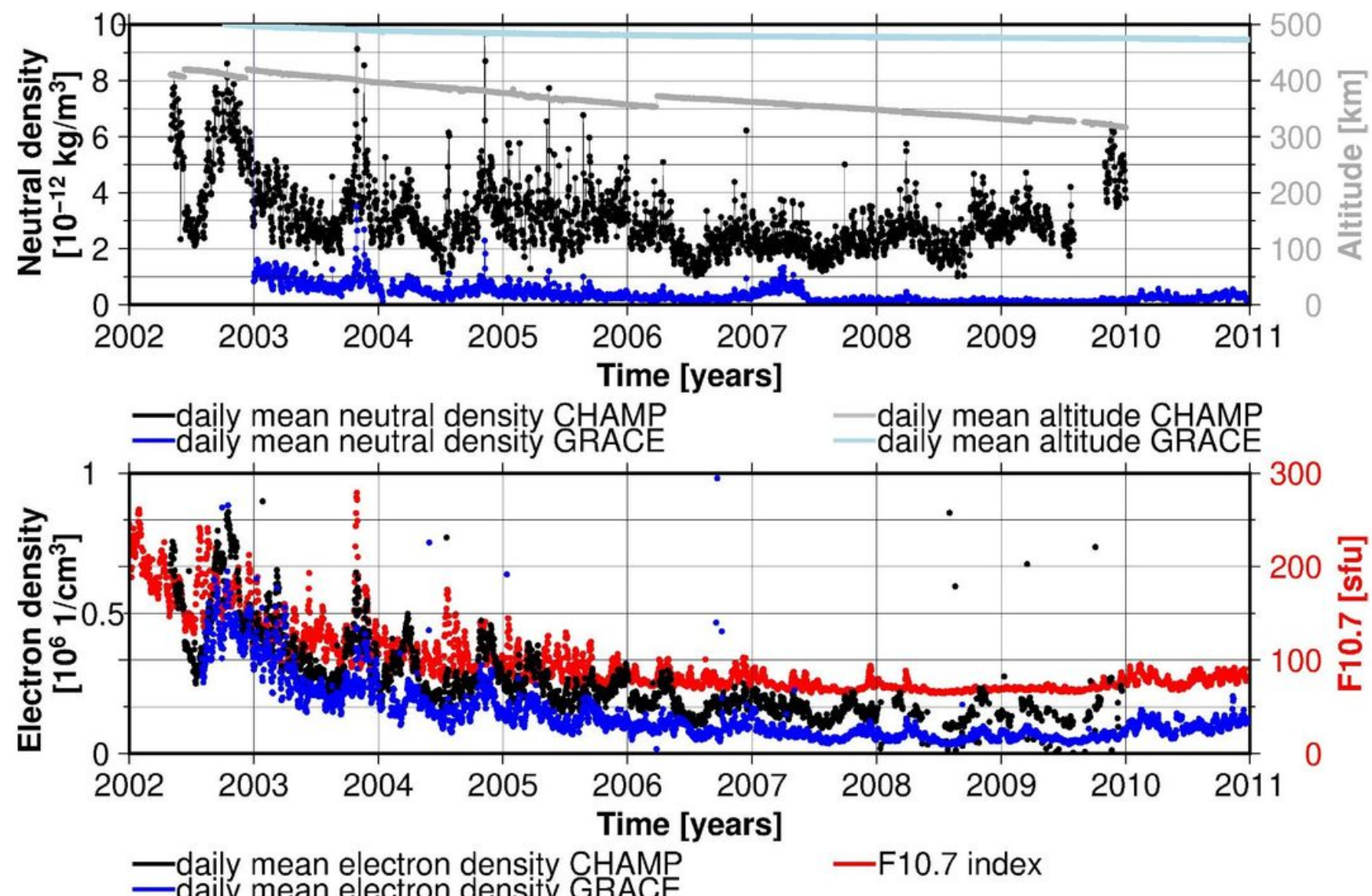

Figure 1

Daily mean neutral (top) and electron (bottom) density of the satellites CHAMP (black) and GRACE (blue) between 2002 and 2010, together with the F10.7 index (red) and daily mean altitudes (light colors). 

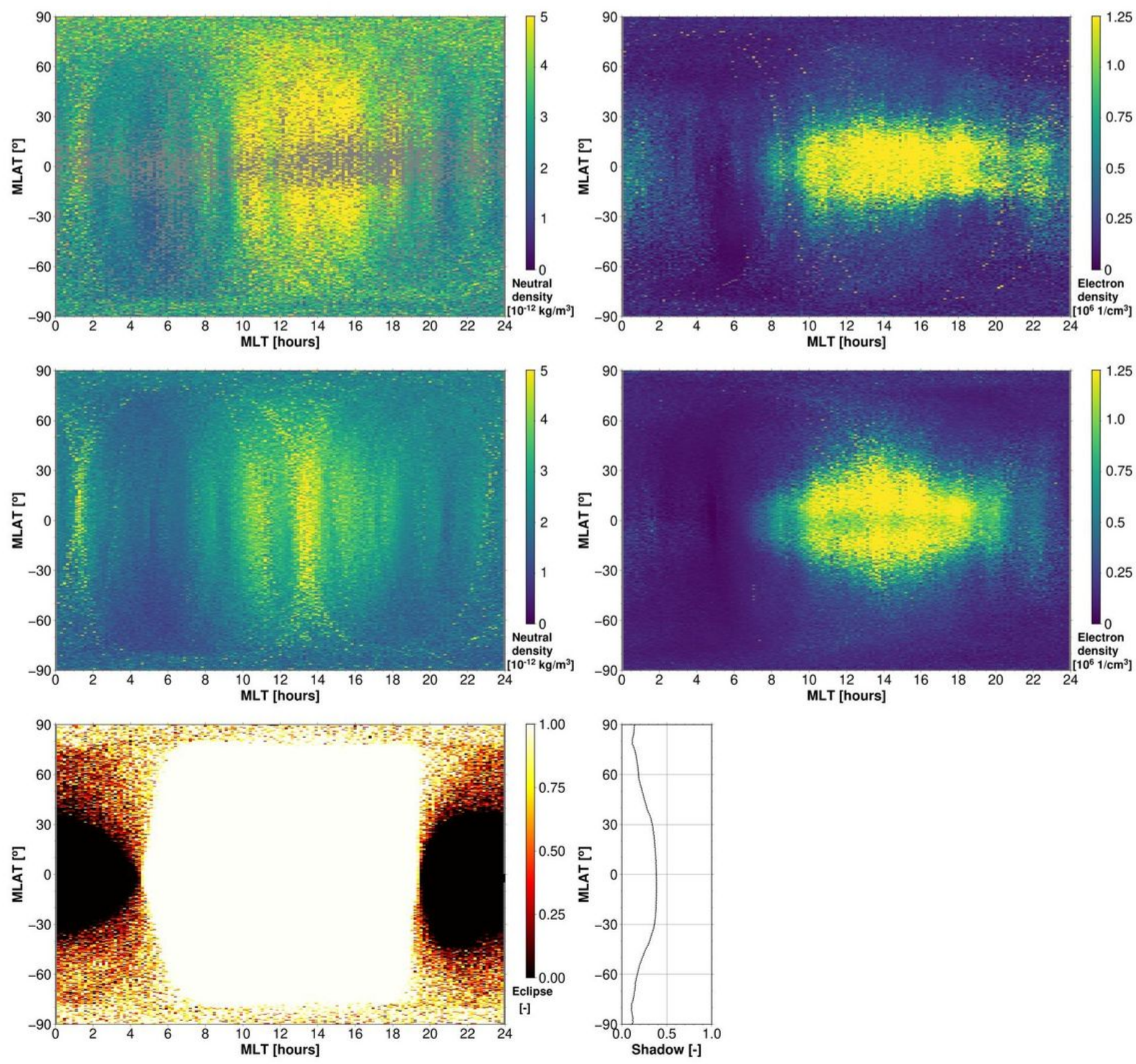

\section{Figure 2}

CHAMP neutral density (left) and electron density (right) for the whole year 2003. Data from measurements (top), modeled from TIE-GCM (middle). Note for neutral densities: gaps below 200 seconds are interpolated in all data sets. Remaining gaps (grey) in the measured neutral densities (top, left) are due to missing measurements. Bottom: Shadow function along the orbit of the CHAMP satellite for the whole year 2003 and the amount of shadow per MLAT. 


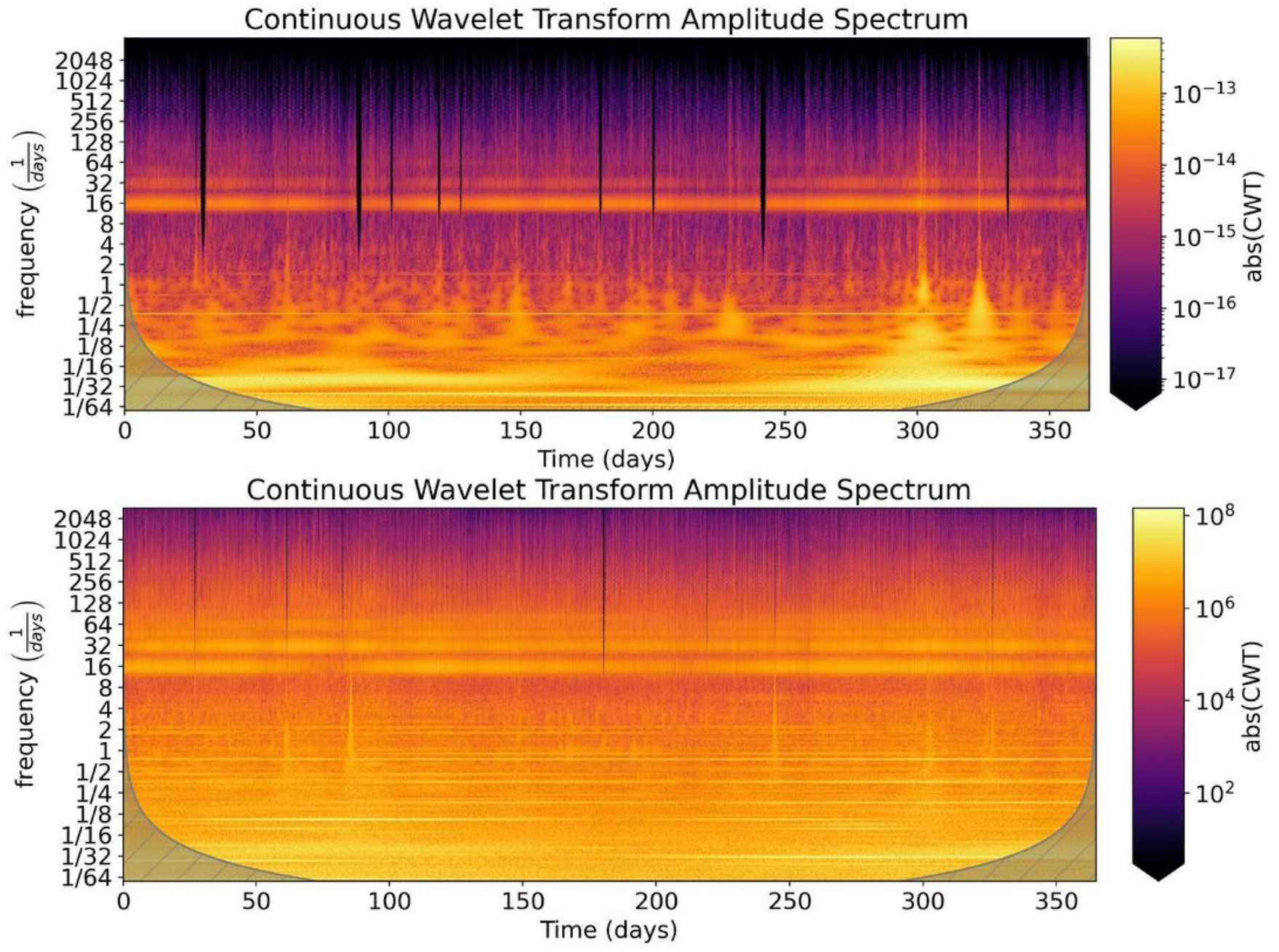

Figure 3

Wavelet analysis of CHAMP accelerometer-derived neutral densities (top) and electron densities from PLP data (bottom) for the whole year 2003 

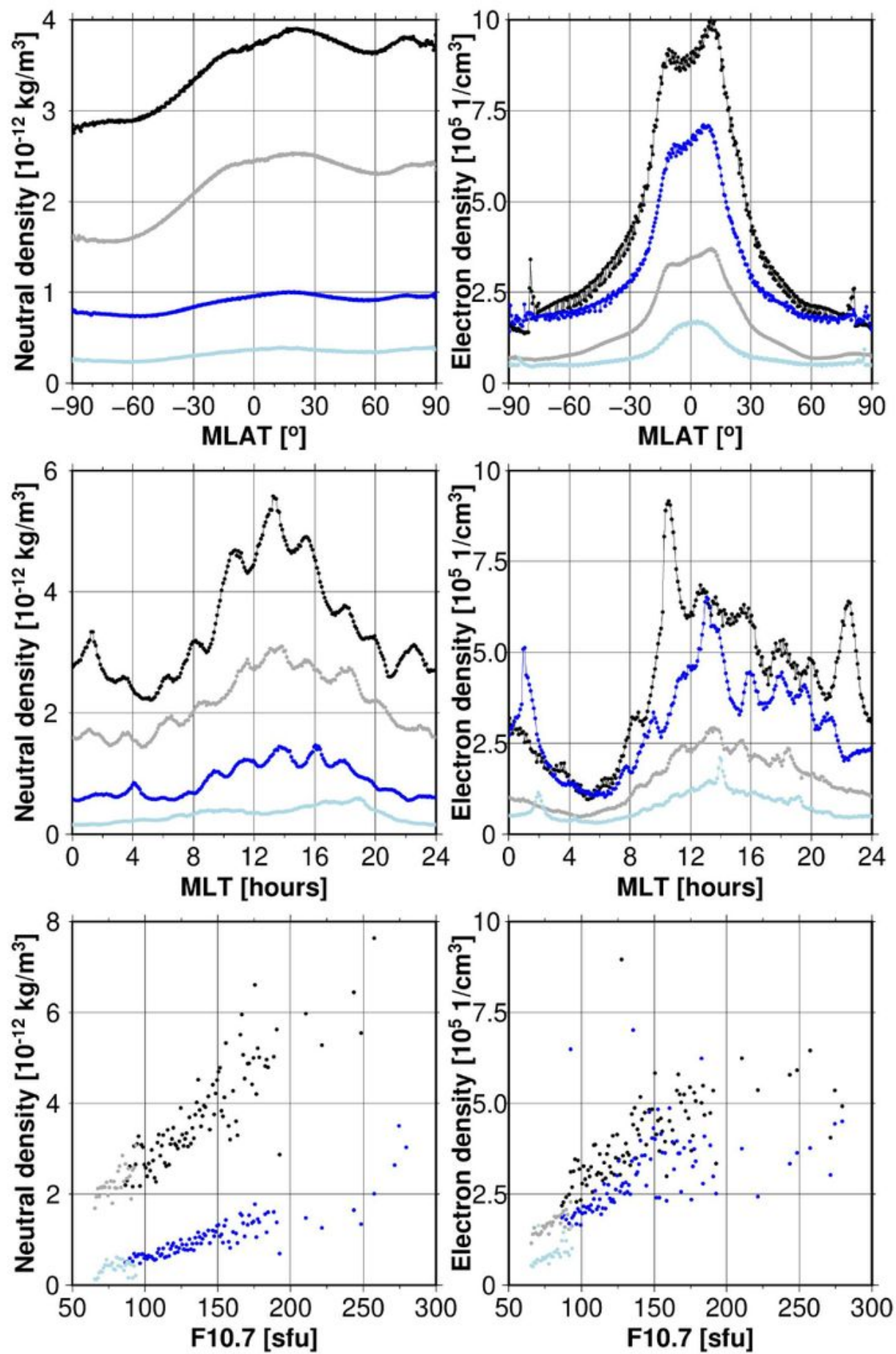

—CHAMP 2003 -CHAMP 2007 —GRACE 2003 -GRACE 2007

\section{Figure 4}

Dependencies of neutral densities (left) and electron densities (right) averaged per magnetic latitude (top), magnetic local time (middle) and daily F10.7 index (bottom) 

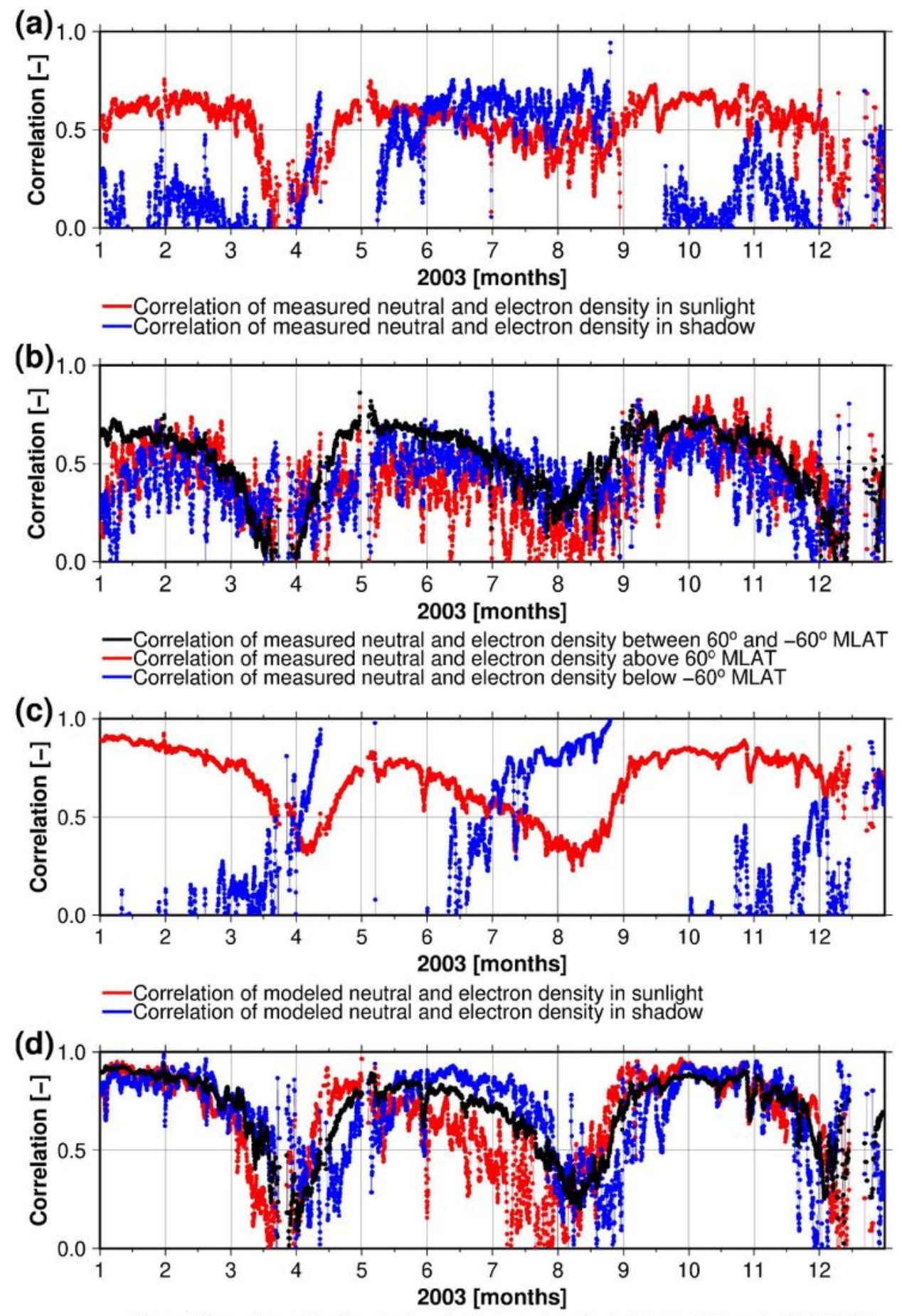

-Correlation of modeled neutral and electron density between $60^{\circ}$ and $-60^{\circ} \mathrm{MLAT}$ -Correlation of modeled neutral and electron density above $60^{\circ} \mathrm{MLAT}$

\section{Figure 5}

Correlation analysis of measured ( $a, b)$ and modeled (c, d) CHAMP neutral and electron densities for the whole year 2003. a, c: The correlation coefficients have been computed from the available densities in sunlight or shadow per orbit, then the correlations from 15 orbits were averaged. b, d: Same, but with data only at specific magnetic latitudes. 

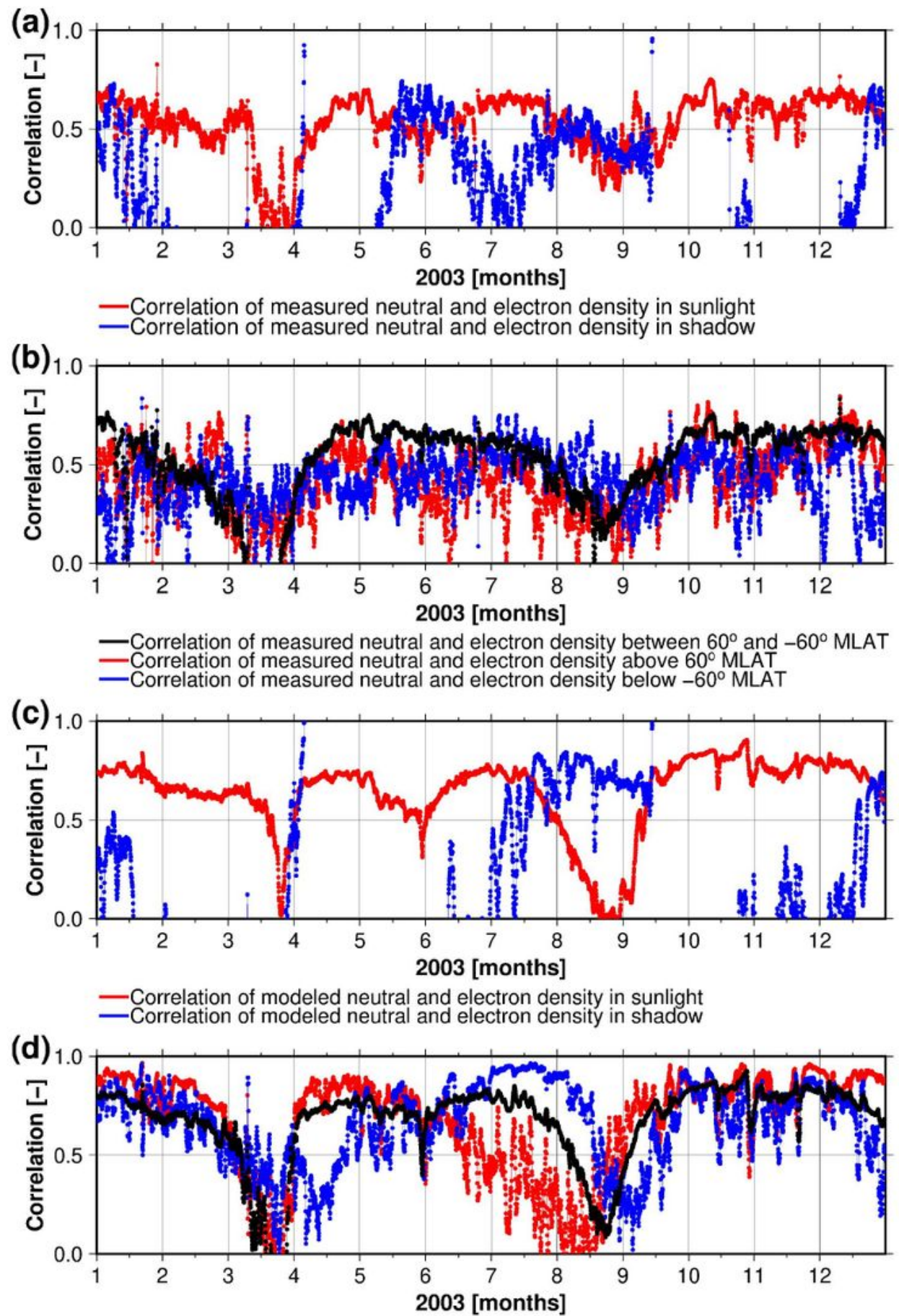

- Correlation of modeled neutral and electron density between $60^{\circ}$ and $-60^{\circ} \mathrm{MLAT}$ -Correlation of modeled neutral and electron density above $60^{\circ} \mathrm{MLAT}$

Figure 6

Correlation analysis of measured $(a, b)$ and modeled (c, d) GRACE neutral and electron densities for the whole year 2003. a, c: The correlation coefficients have been computed from the available densities in sunlight or shadow per orbit, then the correlations from 15 orbits were averaged. b, d: Same, but with data only at specific magnetic latitudes. 
(a)
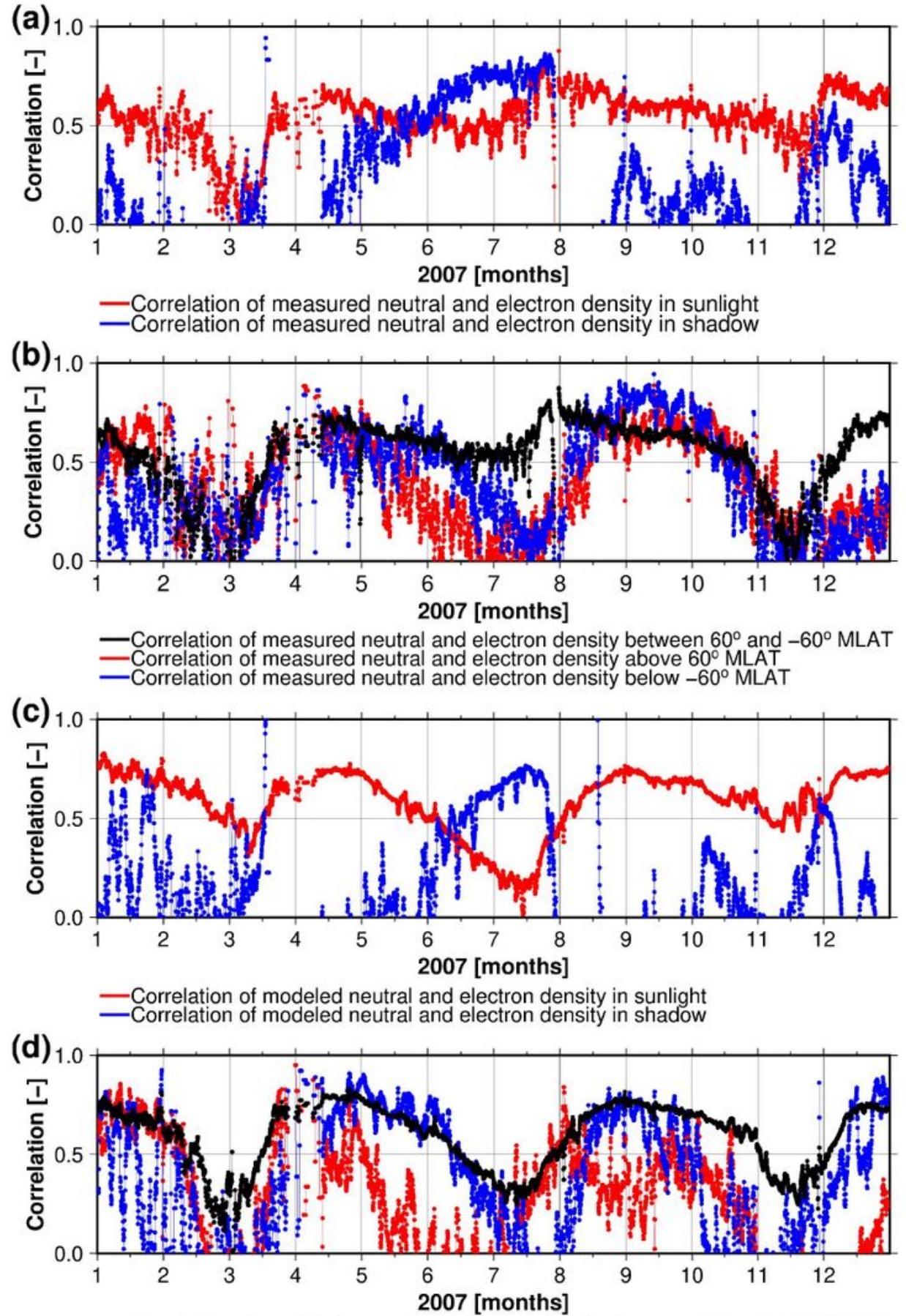

- Correlation of modeled neutral and electron density between $60^{\circ}$ and $-60^{\circ} \mathrm{MLAT}$ -Correlation of modeled neutral and electron density above $60^{\circ} \mathrm{MLAT}$

\section{Figure 7}

Correlation analysis of measured ( $a, b)$ and modeled (c, d) CHAMP neutral and electron densities for the whole year 2007. a, c: The correlation coefficients have been computed from the available densities in sunlight or shadow per orbit, then the correlations from 15 orbits were averaged. b, d: Same, but with data only at specific magnetic latitudes 

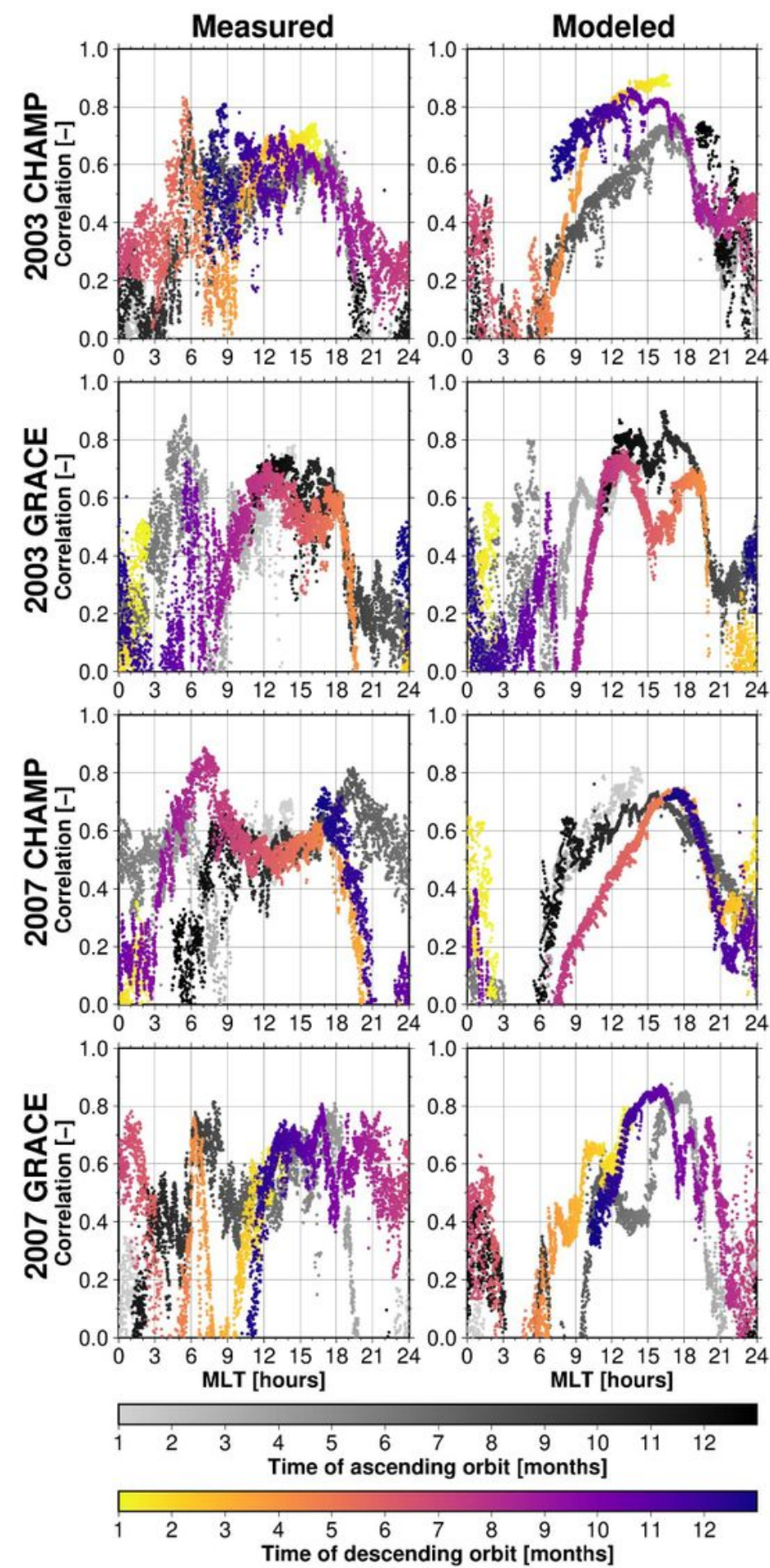

\section{Figure 8}

Correlation coefficients of neutral and electron densities with respect to magnetic latitude for measured data (left column) and modeled data (right column) for GRACE and CHAMP during the years 2003 and 2007. The correlation coefficients have been averaged over 15 ascending and 15 descending orbits, respectively. 


\section{Supplementary Files}

This is a list of supplementary files associated with this preprint. Click to download.

- 01tiegcm2.0res2.52003steadystate.inp

- 02tiegcm2.0res2.52003beforehalloween.inp

- 03tiegcm2.0res2.52003halloween.inp

- 04tiegcm2.0res2.52003afterhalloween.inp

- 05tiegcm2.0res2.52007steadystate.inp

- 06tiegcm2.0res2.52007.inp

- graphicalabstract.png 